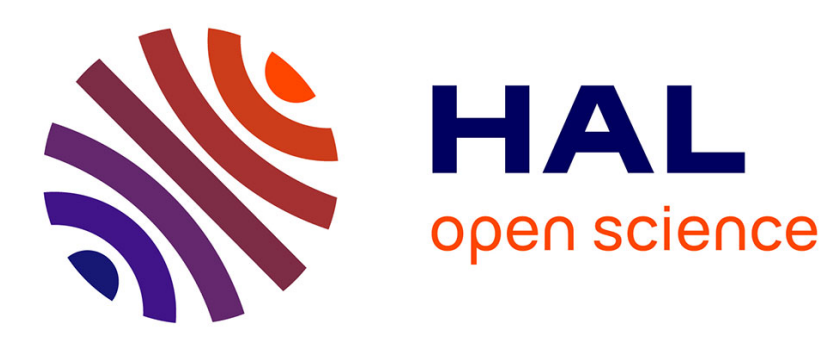

\title{
From Hermite to stationary subdivision schemes in one and several variables
}

\author{
Jean-Louis Merrien, Tomas Sauer
}

\section{To cite this version:}

Jean-Louis Merrien, Tomas Sauer. From Hermite to stationary subdivision schemes in one and several variables. Advances in Computational Mathematics, 2012, 36 (4), pp.547-579. 10.1007/s10444-0119190-7 . hal-00468245

\section{HAL Id: hal-00468245 \\ https://hal.science/hal-00468245}

Submitted on 30 Mar 2010

HAL is a multi-disciplinary open access archive for the deposit and dissemination of scientific research documents, whether they are published or not. The documents may come from teaching and research institutions in France or abroad, or from public or private research centers.
L'archive ouverte pluridisciplinaire HAL, est destinée au dépôt et à la diffusion de documents scientifiques de niveau recherche, publiés ou non, émanant des établissements d'enseignement et de recherche français ou étrangers, des laboratoires publics ou privés. 


\title{
From Hermite to Stationary Subdivision Schemes in One and Several Variables
}

\author{
Jean-Louis Merrien* Tomas Sauer ${ }^{\dagger}$
}

March 30, 2010

\begin{abstract}
Vector and Hermite subdivision schemes both act on vector data, but since the latter one interprets the vectors as function values and consecutive derivatives they differ by the "renormalization" of the Hermite scheme in any step. In this paper we give an algebraic factorization method in one and several variables to relate any Hermite subdivision scheme that satisfies the so-called spectral condition to a vector subdivision scheme. These factorizations are natural extensions of the "zero at $\pi$ " condition known for the masks of refinable functions. Moreover, we show how this factorization can be used to investigate different forms of convergence of the Hermite scheme and why the multivariate situation is conceptionally more intricate than the univariate one. Finally, we give some examples of such factorizations.
\end{abstract}

Keywords: Subdivision, Hermite, Taylor expansion, Factorization.

AMS Subject classification: 41A60, 65D15, $13 \mathrm{P} 05$.

\section{Introduction}

Subdivision algorithms are iterative methods for producing curves and surfaces with a built-in multiresolution structure. They are now used in curve and surface modelling in computer-aided geometric design, video games, animation and many other applications. Stationary and homogeneous subdivision schemes iterate the same subdivision operator and use rules that, independently of the location, compute new values of a refined discrete sequence only from a certain amount of local and neighboring data. This data can be scalar or vector even matrix valued, the matrix case been done by doing vector schemes columnwise, and the stationary, i.e. local and neighboring rule is always acting on a vector sequence $c$ as

$$
S_{\boldsymbol{A}} \boldsymbol{c}=\sum_{\alpha \in \mathbb{Z}^{s}} \boldsymbol{A}(\cdot-2 \alpha) \boldsymbol{c}(\alpha)
$$

\footnotetext{
${ }^{*}$ J.-L. Merrien, INSA de Rennes, 20 av. des Buttes de Coesmes, CS 14315, 35043 RENNES CEDEX, France, email: Jean-Louis.Merrien@insa-rennes.fr

${ }^{\dagger}$ T. Sauer, Lehrstuhl für Numerische Mathematik, Justus-Liebig-Universität Gießen, Heinrich-BuffRing 44, D-35392 Gießen, Germany, email: Tomas.Sauer@math.uni-giessen.de
} 
where the finitely supported sequence of coefficients is referred to as the mask of the subdivision scheme. This operator is iterated, leading to a sequence $S_{A}^{n} c, n \in \mathbb{N}$, of vector valued sequences, which, when related to the finer and finer grid $2^{-n} \mathbb{Z}^{s}$, converges to a limit vector field with certain properties. Such schemes have been investigated for example in $[4,13,16,20,21]$ and there exists quite a substantial amount of literature on vector subdivision schemes in one and several variables meanwhile, investing convergence as well as smoothness of the limit functions or the multiresolution structures generated by these functions.

If the vector data to be processed represents the value and consecutive differences of a function in the so-called Hermite subdivision schemes, the situation changes significantly due to the particular meaning of the vector's coefficients. Hermite subdivision schemes have also been studied by many authors, for example, [5, 11, 12, 17, 19]. As a consequence, a Hermite subdivision scheme is a so called non stationary scheme with a mask at step $n$ given by $\boldsymbol{A}_{n}=\boldsymbol{D}^{-n-1} \boldsymbol{A} \boldsymbol{D}^{n}$, see (6), where $\boldsymbol{A}$ is a usual mask and $\boldsymbol{D}$ is a diagonal matrix with diagonal entries $2^{-j}, j \in\{0, \ldots, d\}$ coming from the derivatives of $\psi(\cdot)=\phi(2 \cdot)$. This matrix creates a new problem since the successive powers of $\boldsymbol{D}^{-1}$ have no limit when $n$ goes to $\infty$ and almost all the components of $\boldsymbol{D}^{n}$ goes to 0 . So it is interesting to transform a Hermite subdivision scheme into a Stationary Subdivision Scheme using a Taylor operator which comes from the usual Taylor expansion.

\section{Notation and basic concepts}

Vectors in $\mathbb{R}^{r}$ will be labeled by lowercase boldface letters as $\boldsymbol{y}=\left[y_{j}\right]_{j=1, \ldots, r}$. In particular, $\boldsymbol{e}_{j}, j=1, \ldots, r$, stands for the canonical unit vectors for which $\left(\boldsymbol{e}_{j}\right)_{k}=$ $e_{j k}=\delta_{j k}, k=1, \ldots, r$, holds true. Matrices in $\mathbb{R}^{r \times r}$ will be written as uppercase boldface letters, like $\boldsymbol{A}=\left[a_{j k}\right]_{j, k=1, \ldots, r}$.

By $\boldsymbol{A} \in \ell^{r \times r}\left(\mathbb{Z}^{s}\right)$, we also denote a multiindexed sequence of matrices, that is, for all $\alpha \in \mathbb{Z}^{s}$ the sequence element $\boldsymbol{A}(\alpha)=\left[a_{j k}(\alpha)\right]_{j, k=1, \ldots . r} \in \mathbb{R}^{r \times r}$ is an $r \times r$ matrix. Any such sequence will be called a mask provided that it is finitely supported, that is, there exists $N \in \mathbb{N}$ such that

$$
\operatorname{supp} \boldsymbol{A}:=\left\{\alpha \in \mathbb{Z}^{s}: \boldsymbol{A}(\alpha) \neq \mathbf{0}\right\} \subseteq[-N, N]^{s} .
$$

To any mask $\boldsymbol{A}$ we associate the stationary subdivision operator $S_{\boldsymbol{A}}: \ell^{r}\left(\mathbb{Z}^{s}\right) \rightarrow$ $\ell^{r}\left(\mathbb{Z}^{s}\right)$, defined as

$$
S_{\boldsymbol{A}} \boldsymbol{c}(\alpha):=\sum_{\beta \in \mathbb{Z}^{s}} \boldsymbol{A}(\alpha-2 \beta) \boldsymbol{c}(\beta), \quad \boldsymbol{c} \in \ell^{r}\left(\mathbb{Z}^{s}\right) .
$$

Polynomials and Laurent polynomials play a fundamental role in the study of subdivision schemes, so we recall the definitions of $\Pi=\mathbb{R}[x]=\mathbb{R}\left[x_{1}, \ldots, x_{s}\right]$ and $\Lambda=\mathbb{R}\left[x, x^{-1}\right]$, respectively, where, using the monomials $x^{\alpha}:=x_{1}^{\alpha_{1}} \ldots x_{s}^{\alpha_{s}}$,

$$
f(x)=\sum_{\alpha \in \mathbb{N}_{0}^{s}} f_{\alpha} x^{\alpha} \in \Pi, \quad f(x)=\sum_{\alpha \in \mathbb{Z}^{s}} f_{\alpha} x^{\alpha} \in \Lambda,
$$


but in both cases only finitely many of the coefficients $f_{\alpha}$ are different from zero so that we are dealing with finite sums in (2). Though polynomials and Laurent polynomials appear to be almost the same - after all, any Laurent polynomial is obtained by multiplying a polynomial of the form $x^{\alpha}, \alpha \in \mathbb{Z}^{s}$, which is a unit in $\Lambda$ - they are of surprisingly different algebraic structure, cf. [22]. By $\Pi_{k}$ we denote the finite dimensional vector space of all polynomials of total degree at most $k$, that is

$$
f(x)=\sum_{|\alpha| \leq k} f_{\alpha} x^{\alpha} \in \Pi_{k}, \quad|\alpha|:=\sum_{j=1}^{s} \alpha_{j}, \quad \alpha \in \mathbb{N}_{0}^{s},
$$

where $\operatorname{dim} \Pi_{k}=r_{k}:=\left(\begin{array}{c}k+s \\ s\end{array}\right)$.

Recall that $s$ denotes the number of variables and write, for $k \in \mathbb{N}, s_{k}:=\left(\begin{array}{c}s+k-1 \\ s-1\end{array}\right)$ for the dimension of the space

$$
\Pi_{k}^{0}:=\operatorname{span}\left\{x^{\alpha}:|\alpha|=k\right\}
$$

of homogeneous polynomials of degree $k$. Obviously, the dimension $r_{k}$ of $\Pi_{k}=$ $\bigoplus_{j=0}^{k} \Pi_{k}^{0}$ satisfies $r_{k}=s_{0}+\cdots+s_{k}$.

A convenient way of studying masks, well used in signal processing, is to consider its symbol which is the - in our case matrix valued - Laurent polynomial

$$
\boldsymbol{A}^{*}(z):=\sum_{\alpha \in \mathbb{Z}^{s}} \boldsymbol{A}(\alpha) z^{\alpha}, \quad z \in \mathbb{C}_{\times}^{s}, \quad \mathbb{C}_{\times}=\mathbb{C} \backslash\{0\} .
$$

Moreover, $\boldsymbol{A}^{*}\left(z^{-1}\right)$ is the well-known $z$-transform from signal processing, cf. [14, 15, 28]. In particular, for $c \in \ell^{r}\left(\mathbb{Z}^{s}\right)$ we have the identity

$$
\left(S_{\boldsymbol{A}} \boldsymbol{c}\right)^{*}(z)=\boldsymbol{A}^{*}(z) \boldsymbol{c}^{*}\left(z^{2}\right) .
$$

If $\epsilon_{j}$ is the $j$-th vector of the canonical basis in $\mathbb{R}^{s}$, we will use the forward difference operators $\Delta_{j}, j=1, \ldots, s$, defined by $\Delta_{j} \boldsymbol{c}=\boldsymbol{c}\left(\cdot+\epsilon_{j}\right)-\boldsymbol{c}$ and the difference operator

$$
\Delta: \ell^{r}\left(\mathbb{Z}^{s}\right) \rightarrow \ell^{s r}\left(\mathbb{Z}^{s}\right), \quad \Delta c=\left[\begin{array}{c}
\Delta_{1} c \\
\vdots \\
\Delta_{s} c
\end{array}\right]
$$

which plays the role of a discrete gradient.

We will first state and prove the Taylor factorization in one variable in Section 3 to explain its basic idea and then turn to the notationally and conceptionally more intricate multivariate situation later (Section 4).

One key argument is the following factorization result: If $a \in \ell\left(\mathbb{Z}^{s}\right)$, satisfies, for all $\alpha \in \mathbb{Z}^{s}$, the condition $\sum_{\beta \in \mathbb{Z}^{s}} a(\alpha-2 \beta)=0$, then there exist $b_{j} \in \ell\left(\mathbb{Z}^{s}\right)$, $j=1, \ldots, s$, such that

$$
a^{*}(z)=\sum_{j=1}^{s}\left(z_{j}^{-2}-1\right) b_{j}^{*}(z)
$$


see $[1,23]$ for a proof. This is a generalization of the well known result in one dimension, $s=1$, that

$$
\sum_{\beta \in \mathbb{Z}} a(\alpha-2 \beta)=0, \quad \alpha \in \mathbb{Z}, \quad \Rightarrow \quad a^{*}(z)=\left(z^{-2}-1\right) b^{*}(z) .
$$

Now we define the Hermite subdivision scheme $H_{A}$. In dimension $s$ with $d$ derivatives, starting with $\boldsymbol{f}_{0} \in \ell^{r_{d}}\left(\mathbb{Z}^{s}\right)$, for $n=0, \ldots$, we define $\boldsymbol{f}_{n+1} \in \ell^{r_{d}}\left(\mathbb{Z}^{s}\right)$ by

$$
\boldsymbol{D}^{n+1} \boldsymbol{f}_{n+1}(\alpha)=\sum_{\beta \in \mathbb{Z}^{s}} \boldsymbol{A}(\alpha-2 \beta) \boldsymbol{D}^{n} \boldsymbol{f}_{n}(\beta), \quad \boldsymbol{c} \in \ell^{r_{d}}\left(\mathbb{Z}^{s}\right),
$$

where

$$
\boldsymbol{D}=\left[\begin{array}{llll}
1 & & & \\
& \frac{1}{2} \boldsymbol{I}_{s_{1}} & & \\
& & \ddots & \\
& & & \frac{1}{2^{d}} \boldsymbol{I}_{s_{d}}
\end{array}\right]
$$

is the diagonal matrix with diagonal entries $2^{-j}$ repeated $s_{j}$ times, respectively, $j=$ $0, \ldots, d$.

For the iterated application of the Hermite subdivision scheme, we decompose the vector into the block form

$$
\boldsymbol{f}_{n}(\beta)=\left[\boldsymbol{f}_{n}^{(j)}(\beta)\right]_{j=0, \ldots, d}, \quad \beta \in \mathbb{Z}^{s}, \quad \boldsymbol{f}_{n}^{(j)} \in \ell^{s_{j}}\left(\mathbb{Z}^{s}\right),
$$

and the first component $\boldsymbol{f}_{n}^{(0)}(\beta)$ can be interpreted as the value of a function $\phi_{n}$ at $\beta / 2^{n}$, while the $s_{1}$ following ones, $\boldsymbol{f}_{n}^{(1)}(\beta)$, describe the gradient or first total derivative $D^{1} \phi_{n}\left(\beta / 2^{n}\right)$, and so on, up to the last $s_{d}$ ones, $\boldsymbol{f}_{n}^{(d)}(\beta)$ which are $D^{d} \phi_{n}\left(\beta / 2^{n}\right)$, where $D^{j}:=\left[D^{\alpha}=\frac{\partial^{j}}{\partial x^{\alpha}}\right]_{|\alpha|=j}, j=0, \ldots, d$.

The main goal of this paper is to prove that, under suitable hypotheses, namely the spectral condition, a Hermite subdivision operator can be transformed into an equivalent stationary subdivision operator which we will call the Taylor subdivision operator associated to $H_{\boldsymbol{A}}$ (Section 3 for the dimension $s=1$ and Section 4 for $s>1$ ).

Then, in Section 5, for $s=1$, we will prove that if the Taylor subdivision operator is convergent as a stationary vector subdivision scheme, then $H_{\boldsymbol{A}}$ is convergent in the sense of Hermite subdivision schemes. For $s>1$, when adding further hypothesis, we will give a generalization of the previous results.

In the last Section, we give examples of interpolatory and non interpolatory schemes and their transformation into stationary subdivision schemes.

\section{The Taylor factorization in one variable, $s=1$}

We will use a function and its $d$ derivatives, but since $s=1$, we are in the special situation that, for any $k \in \mathbb{N}, s_{k}=1$ and $r=r_{d}=d+1$.

In this section, we will show that any subdivision operator that satisfies the spectral condition will admit a factorization in terms of operators. In fact, this result was 
already proved in [10]. We give a new proof using different operators and a more algebraic approach.

To introduce the definition of the spectral condition, we associate to any function $f \in C^{d}(\mathbb{R})$ the vector sequence $\boldsymbol{v}_{f} \in \ell^{d+1}(\mathbb{Z})$ with

$$
\boldsymbol{v}_{f}(\alpha):=\left[\begin{array}{c}
f(\alpha) \\
f^{\prime}(\alpha) \\
\vdots \\
f^{(d)}(\alpha)
\end{array}\right], \quad \alpha \in \mathbb{Z} .
$$

Definition 1 A mask $\boldsymbol{A}$ or its associated subdivision operator $S_{\boldsymbol{A}}$ satisfies the spectral condition of order $d^{\prime} \leq d$ if there exist polynomials $p_{j} \in \Pi_{j}$, $\operatorname{deg} p_{j}=j, j=0, \ldots, d^{\prime}$, such that

$$
S_{\boldsymbol{A}} \boldsymbol{v}_{j}=\frac{1}{2^{j}} \boldsymbol{v}_{j}, \quad \boldsymbol{v}_{j}:=\boldsymbol{v}_{p_{j}}=\left[\begin{array}{c}
p_{j} \\
p_{j}^{\prime} \\
\vdots \\
p_{j}^{(d)}
\end{array}\right],
$$

where we will always assume that $p_{j}$ is normalized such that $p_{j}(x)=\frac{1}{j !} x^{j}+\cdots$.

It was proved in [10] that the spectral condition is also equivalent to the sum rule introduced by Bin Han et al [16, 17].

Definition 2 The Taylor operator $T_{d}$ of order d, acting on $\ell^{d+1}(\mathbb{Z})$, is defined as

$$
T_{d}:=\left[\begin{array}{ccccc}
\Delta & -1 & \ldots & -\frac{1}{(d-1) !} & -\frac{1}{d !} \\
& \Delta & \ddots & \vdots & \vdots \\
& & \ddots & -1 & \vdots \\
& & & \Delta & -1 \\
& & & & 1
\end{array}\right] .
$$

The name "Taylor operator" is easily explained: If $f \in C^{d}(\mathbb{R})$ then, for any $\alpha \in \mathbb{Z}$,

$$
\left(T_{d} \boldsymbol{v}_{f}\right)_{j}(\alpha)=f^{(j)}(\alpha+1)-\sum_{k=0}^{d-j} \frac{1}{k !} f^{(j+k)}(\alpha), \quad j=0, \ldots, d-1,
$$

are precisely the terms appearing in the Taylor expansion of $f$, as well as $\left(T_{d} \boldsymbol{v}_{f}\right)_{d}(\alpha)=$ $f^{(d)}(\alpha)$.

Remark 3 An alternative version of the Taylor operator, namely

$$
T_{d}^{\prime}:=\left[\begin{array}{ccccc}
\Delta & -1 & \ldots & -\frac{1}{(d-1) !} & 0 \\
& \Delta & \ddots & & \vdots \\
& & \ddots & -1 & \vdots \\
& & & \Delta & 0 \\
& & & & 1
\end{array}\right]
$$

was introduced and investigated in [10]. 
To transform the Hermite subdivision scheme into a stationary subdivision scheme, we will also need a slight modification of the Taylor operator from (9), namely the complete Taylor operator acting on $\ell^{(d+1)}(\mathbb{Z})$

$$
\widetilde{T}_{d}:=\left[\begin{array}{ccccc}
\Delta & -1 & \ldots & -\frac{1}{(d-1) !} & -\frac{1}{d !} \\
& \Delta & \ddots & \vdots & \vdots \\
& & \ddots & -1 & \vdots \\
& & & \Delta & -1 \\
& & & & \Delta
\end{array}\right]
$$

We notice that $\widetilde{T}_{0}^{*}(z)=z^{-1}-1$ and for $k=1, \ldots, d$,

$$
\widetilde{T}_{k+1}^{*}(z)=\left[\begin{array}{cc}
\widetilde{T}_{k}^{*}(z) & -\boldsymbol{w}_{k} \\
\mathbf{0} & z^{-1}-1
\end{array}\right], \quad \boldsymbol{w}_{k}:=\left[\begin{array}{c}
\frac{1}{(k+1) !} \\
\frac{1}{k !} \\
\vdots \\
1
\end{array}\right] .
$$

To simplify notation, we will write $\boldsymbol{I}$ and $\mathbf{0}$ for identity and zero matrices of appropriate size whenever their dimensions are clear from the context.

Theorem 4 If the mask $\boldsymbol{A} \in \ell^{(d+1) \times(d+1)}(\mathbb{Z})$ satisfies the spectral condition of order $d$, then there exist two finitely supported masks $\boldsymbol{B}, \widetilde{\boldsymbol{B}} \in \ell^{(d+1) \times(d+1)}(\mathbb{Z})$ such that

$$
T_{d} S_{\boldsymbol{A}}=2^{-d} S_{\boldsymbol{B}} T_{d} \quad \text { and } \quad \widetilde{T}_{d} S_{\boldsymbol{A}}=2^{-d} S_{\widetilde{B}} \widetilde{T}_{d} .
$$

We will split the proof of Theorem 4 into several pieces, beginning with a simple observation on $T_{d}$.

Lemma 5 For $p \in \Pi_{d}$, we have that $T_{d} \boldsymbol{v}_{p}=\boldsymbol{e}_{d} p^{(d)}(0)$ and $\widetilde{T}_{d} \boldsymbol{v}_{p}=\mathbf{0}$.

Proof: Making use of (10), we see that for any $\alpha \in \mathbb{Z}$ there exist $\xi_{0}, \ldots, \xi_{d-1} \in(0,1)$ such that

$$
\left(T_{d} \boldsymbol{v}_{p}\right)_{j}(\alpha)=\frac{1}{(d-j+1) !} p^{(d+1)}\left(\alpha+\xi_{j}\right)=0, \quad j=0, \ldots, d-1,
$$

and that $\left(T_{d} \boldsymbol{v}_{p}\right)_{d}(\alpha)=p^{(d)}(\alpha)=p^{(d)}(0)$, since $p \in \Pi_{d}$.

The proof of $\widetilde{T}_{d} \boldsymbol{v}_{p}=\mathbf{0}$ is similar.

Expanding, at first, any $p \in \Pi_{d-1}$ with respect to the basis $\left\{p_{0}, \ldots, p_{d-1}\right\}$, and taking into account that $p_{d}^{(d)}=1$, the following result is an immediate consequence of Lemma 5.

Corollary 6 If the mask $\boldsymbol{A}$ satisfies the spectral condition of order d, then

$$
T_{d} S_{\boldsymbol{A}} \boldsymbol{v}_{p}=0, \quad p \in \Pi_{d-1}, \quad T_{d} S_{\boldsymbol{A}} \boldsymbol{v}_{d}=2^{-d} \boldsymbol{e}_{d}
$$

and $\widetilde{T}_{d} S_{\boldsymbol{A}} \boldsymbol{v}_{p}=0$ for any $p \in \Pi_{d}$. 
Proposition 7 If $S_{\boldsymbol{C}} \boldsymbol{v}_{p}=0$ for all $p \in \Pi_{k}, k \leq d$, then there exists a (finitely supported) mask $\boldsymbol{B}_{k} \in \ell^{(d+1) \times(d+1)}(\mathbb{Z})$ such that

$$
S_{\boldsymbol{C}}=S_{\boldsymbol{B}_{k}}\left[\begin{array}{cc}
\widetilde{T}_{k} & \mathbf{0} \\
\mathbf{0} & \boldsymbol{I}
\end{array}\right]
$$

Proof: Writing $C^{*}(z)=\left[\boldsymbol{c}_{0}^{*}(z) \cdots \boldsymbol{c}_{d}^{*}(z)\right]$ in terms of its column vectors, we shall prove that $S_{\boldsymbol{C}} \boldsymbol{v}_{p}=0, p \in \Pi_{k}$, implies the existence of $\boldsymbol{b}_{0}, \ldots, \boldsymbol{b}_{k}$ such that

$$
\boldsymbol{C}^{*}(z)=\left[\boldsymbol{b}_{0}^{*}(z) \cdots \boldsymbol{b}_{k}^{*}(z) \boldsymbol{c}_{k+1}^{*}(z) \cdots \boldsymbol{c}_{d}^{*}(z)\right]\left[\begin{array}{cc}
\widetilde{T}_{k}^{*}\left(z^{2}\right) & \mathbf{0} \\
\mathbf{0} & \boldsymbol{I}
\end{array}\right]
$$

which defines

$$
\boldsymbol{B}_{k}^{*}(z):=\left[\boldsymbol{b}_{0}^{*}(z) \cdots \boldsymbol{b}_{k}^{*}(z) \boldsymbol{c}_{k+1}^{*}(z) \cdots \boldsymbol{c}_{d}^{*}(z)\right] .
$$

This will be done by induction on $k$.

In the case $k=0$ the assumption implies that $p$ is a constant polynomial, hence

$$
\mathbf{0}=\left(S_{\boldsymbol{C}} \boldsymbol{e}_{0}\right)(\alpha)=\sum_{\beta \in \mathbb{Z}} \boldsymbol{c}_{0}(\alpha-2 \beta),
$$

so that, by (5), there exists $\boldsymbol{b}_{0}^{*}(z)$ such that $\boldsymbol{c}_{0}^{*}(z)=\left(z^{-2}-1\right) \boldsymbol{b}_{0}^{*}(z)$. Consequently,

$$
\begin{aligned}
\boldsymbol{C}^{*}(z) & =\left[\left(z^{-2}-1\right) \boldsymbol{b}_{0}^{*}(z) \boldsymbol{c}_{1}^{*}(z) \cdots \boldsymbol{c}_{d}^{*}(z)\right]=\boldsymbol{B}_{0}^{*}(z)\left[\begin{array}{cc}
z^{-2}-1 & \mathbf{0} \\
\mathbf{0} & \boldsymbol{I}
\end{array}\right] \\
& =\boldsymbol{B}_{0}^{*}(z)\left[\begin{array}{cc}
\widetilde{T}_{0}^{*}\left(z^{2}\right) & \mathbf{0} \\
\mathbf{0} & \boldsymbol{I}
\end{array}\right],
\end{aligned}
$$

which shows the validity of (15) for $k=0$.

Now, let us assume that (14) is satisfied for some $k \geq 0$ and that $S_{\boldsymbol{C}} \boldsymbol{v}_{p}=0$ for all $p \in \Pi_{k+1}$. A simple computation based on (10), like in the proof of Lemma 5, shows that for $p=p_{k+1}$ we get for $\boldsymbol{v}_{p}^{\prime}=\left[\left(\boldsymbol{v}_{p}\right)_{j}\right]_{j=0, \ldots, k} \in \ell^{k+1}(\mathbb{Z})$ that

$$
\widetilde{T}_{k} \boldsymbol{v}_{p}^{\prime}=\boldsymbol{w}_{k} p^{(k+1)}(0)=\boldsymbol{w}_{k},
$$

where $\boldsymbol{w}_{k}$ is defined in (11). By the induction hypothesis and our assumption,

$$
\mathbf{0}=S_{\boldsymbol{C}} \boldsymbol{v}_{p}=S_{\boldsymbol{B}_{k}}\left[\begin{array}{ccc}
\widetilde{T}_{k} & \mathbf{0} & \mathbf{0} \\
\mathbf{0} & 1 & \mathbf{0} \\
\mathbf{0} & \mathbf{0} & \boldsymbol{I}
\end{array}\right]\left[\begin{array}{c}
\boldsymbol{v}_{p}^{\prime} \\
1 \\
\mathbf{0}
\end{array}\right]=S_{\boldsymbol{B}_{k}}\left[\begin{array}{c}
\boldsymbol{w}_{k} \\
1 \\
\mathbf{0}
\end{array}\right]
$$

That is,

$$
\sum_{\beta \in \mathbb{Z}} \sum_{j=0}^{k} \frac{1}{(k+1-j) !} \boldsymbol{b}_{j}(\alpha-2 \beta)+\boldsymbol{c}_{k+1}(\alpha-2 \beta)=0, \quad \alpha \in \mathbb{Z},
$$

and the same argument as before shows that

$$
\sum_{j=0}^{k} \frac{1}{(k+1-j) !} \boldsymbol{b}_{j}^{*}(z)+\boldsymbol{c}_{k+1}^{*}(z)=\boldsymbol{b}_{k+1}^{*}(z)\left(z^{-2}-1\right),
$$


in other words,

$$
\boldsymbol{c}_{k+1}^{*}(z)=\left[\boldsymbol{b}_{0}^{*}(z) \cdots \boldsymbol{b}_{k+1}^{*}(z)\right]\left[\begin{array}{c}
-\boldsymbol{w}_{k} \\
z^{-2}-1
\end{array}\right]
$$

or

$$
\boldsymbol{B}_{k}^{*}(z)=\boldsymbol{B}_{k+1}^{*}(z)\left[\begin{array}{ccc}
\boldsymbol{I} & -\boldsymbol{w}_{k} & \mathbf{0} \\
\mathbf{0} & z^{-2}-1 & \mathbf{0} \\
\mathbf{0} & \mathbf{0} & \boldsymbol{I}
\end{array}\right] .
$$

Substituting this into (15) we thus get

$$
\begin{aligned}
\boldsymbol{C}^{*}(z) & =\boldsymbol{B}_{k}^{*}(z)\left[\begin{array}{cc}
\widetilde{T}_{k}^{*}\left(z^{2}\right) & \mathbf{0} \\
\mathbf{0} & \boldsymbol{I}
\end{array}\right] \\
& =\boldsymbol{B}_{k+1}^{*}(z)\left[\begin{array}{ccc}
\boldsymbol{I} & -\boldsymbol{w}_{k} & \mathbf{0} \\
\mathbf{0} & z^{-2}-1 & \mathbf{0} \\
\mathbf{0} & \mathbf{0} & \boldsymbol{I}
\end{array}\right]\left[\begin{array}{ccc}
\widetilde{T}_{k}^{*}\left(z^{2}\right) & \mathbf{0} & \mathbf{0} \\
\mathbf{0} & 1 & \mathbf{0} \\
\mathbf{0} & \mathbf{0} & \boldsymbol{I}
\end{array}\right] \\
& =\boldsymbol{B}_{k+1}^{*}(z)\left[\begin{array}{ccc}
\widetilde{T}_{k}^{*}\left(z^{2}\right) & -\boldsymbol{w}_{k} & \mathbf{0} \\
\mathbf{0} & z^{-2}-1 & \mathbf{0} \\
\mathbf{0} & \mathbf{0} & \boldsymbol{I}
\end{array}\right]=\boldsymbol{B}_{k+1}^{*}(z)\left[\begin{array}{cc}
\widetilde{T}_{k+1}^{*}\left(z^{2}\right) & \mathbf{0} \\
\mathbf{0} & \boldsymbol{I}
\end{array}\right],
\end{aligned}
$$

which completes the proof.

Corollary 8 For a mask $C \in \ell^{(d+1) \times(d+1)}(\mathbb{Z})$ the following statements are equivalent:

1. $S_{\boldsymbol{C}} \boldsymbol{v}_{p}=\mathbf{0}$ for all $p \in \Pi_{d-1}$,

2. there exists as mask $\boldsymbol{B}^{\prime}$ such that $S_{\boldsymbol{C}}=S_{\boldsymbol{B}^{\prime}} T_{d}^{\prime}$,

3. there exists as mask $\boldsymbol{B}$ such that $S_{\boldsymbol{C}}=S_{\boldsymbol{B}} T_{d}$.

Proof: By Proposition 7,

$$
\boldsymbol{C}^{*}(z)=\boldsymbol{B}_{d-1}^{*}(z)\left[\begin{array}{cc}
\widetilde{T}_{d-1}\left(z^{2}\right) & \mathbf{0} \\
\mathbf{0} & 1
\end{array}\right]=\boldsymbol{B}_{d-1}^{*}(z) T_{d}^{\prime}\left(z^{2}\right),
$$

hence, $\boldsymbol{B}^{\prime}=\boldsymbol{B}_{d-1}$ satisfies 2). To prove 3), we expand the above identity into

$$
\begin{aligned}
\boldsymbol{C}^{*}(z) & =\boldsymbol{B}_{d-1}^{*}(z)\left[\begin{array}{cc}
\boldsymbol{I} & \boldsymbol{w} \\
\mathbf{0} & 1
\end{array}\right]\left[\begin{array}{cc}
\boldsymbol{I} & -\boldsymbol{w} \\
\mathbf{0} & 1
\end{array}\right] T_{d}^{\prime}\left(z^{2}\right) \\
& =\boldsymbol{B}_{d-1}^{*}(z)\left[\begin{array}{cc}
\boldsymbol{I} & \boldsymbol{w} \\
\mathbf{0} & 1
\end{array}\right] T_{d}\left(z^{2}\right)=: \boldsymbol{B}^{*}(z) T_{d}\left(z^{2}\right) .
\end{aligned}
$$

Conversely, since for $p \in \Pi_{d-1}$ we have that $T_{d}^{\prime} \boldsymbol{v}_{p}=T_{d} \boldsymbol{v}_{p}=\mathbf{0}$, it also follows that $S_{\boldsymbol{A}} \boldsymbol{v}_{p}=S_{\boldsymbol{B}^{\prime}} T_{d}^{\prime} \boldsymbol{v}_{p}=S_{\boldsymbol{B}} T_{d} \boldsymbol{v}_{p}=\mathbf{0}$ for $p \in \Pi_{d-1}$.

Corollary 9 A mask $\boldsymbol{C} \in \ell^{(d+1) \times(d+1)}(\mathbb{Z})$ satisfies $S_{\boldsymbol{C}} \boldsymbol{v}_{p}=0$ for all $p \in \Pi_{d}$ if and only if there exists a mask $\widetilde{B}$ such that $S_{\boldsymbol{C}}=S_{\widetilde{\boldsymbol{B}}} \widetilde{T}_{d}$. 
With these observations in hand, the proof of Theorem 4 is now easily completed.

Proof of Theorem 4: Defining $\boldsymbol{C}$ by $\boldsymbol{C}^{*}(z)=2^{d} \boldsymbol{T}_{d}^{*}(z) \boldsymbol{A}^{*}(z)$ and noting that for $p=p_{k} \in \Pi_{k}, k=0, \ldots, d-1$, we get that

$$
S_{\boldsymbol{C}} \boldsymbol{v}_{k}=T_{d} S_{\boldsymbol{A}} \boldsymbol{v}_{k}=\frac{1}{2^{k}} T_{d} \boldsymbol{v}_{k}=\mathbf{0}, \quad \boldsymbol{v}_{k}=\boldsymbol{v}_{p_{k}} .
$$

Since $\left\{p_{0}, \ldots, p_{d-1}\right\}$ form a basis of $\Pi_{d-1}$, we have that $S_{\boldsymbol{C}} \boldsymbol{v}_{p}=\mathbf{0}, p \in \Pi_{d-1}$, and Corollary 8 tells us that there exists $\boldsymbol{B}$ such $S_{\boldsymbol{C}}=S_{\boldsymbol{B}} T_{d}$, hence $T_{d} S_{\boldsymbol{A}}=2^{-d} S_{\boldsymbol{B}} T_{d}$ as claimed.

The equality $\widetilde{T}_{d} S_{\boldsymbol{A}}=2^{-d} S_{\widetilde{\boldsymbol{B}}} \widetilde{T}_{d}$ is obtained with $\boldsymbol{C}^{*}(z)=2^{d} \widetilde{\boldsymbol{T}}_{d}^{*}(z) \boldsymbol{A}^{*}(z), k=d$ and $\widetilde{\boldsymbol{B}}=\boldsymbol{B}_{d}$ in Proposition 7 .

\section{The multivariate case}

In the multivariate case, the definition, theorems and proofs follow the same lines as in the univariate situation, but are conceptionally slightly more intricate.

We still consider polynomials of total degree at most $d$ and to any function $f \in$ $C^{d}\left(\mathbb{R}^{s}\right)$ we now associate the vector valued sequence defined as

$$
\boldsymbol{v}_{f}(\gamma):=\left[\begin{array}{c}
f(\gamma) \\
D^{1} f(\gamma) \\
\vdots \\
D^{d} f(\gamma)
\end{array}\right], \quad \gamma \in \mathbb{Z}^{s},
$$

where we recall that $D^{j}:=\left[D^{\alpha}=\frac{\partial^{j}}{\partial x^{\alpha}}\right]_{|\alpha|=j}$ stands for the total differential of order $j$. The partial differences $\Delta_{j}, j=1, \ldots, d$, are now defined as $\Delta_{j} f(\gamma)=f\left(\gamma+\epsilon_{j}\right)-$ $f(\gamma)$ for $\gamma \in \mathbb{Z}^{s}$. Again, we use a partial univariate Taylor expansion in the direction of $\epsilon_{j}$, to observe that for $|\alpha| \leq d$

$$
D^{\alpha} f\left(\gamma+\epsilon_{j}\right)=D^{\alpha} f(\gamma)+\sum_{k=1}^{d-|\alpha|-1} \frac{1}{k !} D^{\alpha+k \epsilon_{j}} f(\gamma)+R f, \quad \gamma \in \mathbb{Z}^{s},
$$

with $R f=0$ if $f \in \Pi_{d-1}$, so that the $j$-th partial Taylor operator operating on $\ell^{r_{d}}\left(\mathbb{Z}^{s}\right)$ takes the form

$$
T_{d}^{j}:=\left[\begin{array}{ccccc}
\Delta_{j} \boldsymbol{I}_{0} & \boldsymbol{T}_{0,1}^{j} & \cdots & \boldsymbol{T}_{0, d-1}^{j} & \boldsymbol{T}_{0, d}^{j} \\
& \Delta_{j} \boldsymbol{I}_{1} & \ddots & \vdots & \vdots \\
& & \ddots & \boldsymbol{T}_{d-2, d-1}^{j} & \vdots \\
& & & \Delta_{j} \boldsymbol{I}_{d-1} & \boldsymbol{T}_{d-1, d}^{j} \\
& & & & \boldsymbol{I}_{d}
\end{array}\right],
$$

where $\boldsymbol{I}_{k} \in \mathbb{R}^{s_{k} \times s_{k}}, k=0, \ldots, d$, are identity matrices of dimension $s_{k} \times s_{k}$ and the matrices $\boldsymbol{T}_{k, \ell}^{j} \in \mathbb{R}^{s_{k} \times s_{\ell}, \ell}=k+1, \ldots, d$, have zero entries except

$$
\left(\boldsymbol{T}_{k, \ell}^{j}\right)_{\alpha, \alpha+(\ell-k) \epsilon_{j}}=-\frac{1}{(\ell-k) !}, \quad|\alpha|=k, \quad \ell=k+1, \ldots, d .
$$


The total Taylor operator is then defined as

$$
T_{d}:=\left[\begin{array}{c}
T_{d}^{1} \\
\vdots \\
T_{d}^{s}
\end{array}\right]
$$

For example, for $s=2$ and $d=2$, we have

$$
\boldsymbol{f}=\left[\begin{array}{c}
f^{(0,0)} \\
f^{(1,0)} \\
f^{(0,1)} \\
f^{(2,0)} \\
f^{(1,1)} \\
f^{(0,2)}
\end{array}\right], T_{2} \boldsymbol{f}=\left[\begin{array}{c}
f^{(0,0)}\left(\cdot+\epsilon_{1}\right)-f^{(0,0)}(\cdot)-f^{(1,0)}(\cdot)-\frac{1}{2} f^{(2,0)}(\cdot) \\
f^{(1,0)}\left(\cdot+\epsilon_{1}\right)-f^{(1,0)}(\cdot)-f^{(2,0)}(\cdot) \\
f^{(0,1)}\left(\cdot+\epsilon_{1}\right)-f^{(0,1)}(\cdot)-f^{(1,1)}(\cdot) \\
f^{(2,0)}(\cdot) \\
f^{(1,1)}(\cdot) \\
f^{(0,2)}(\cdot) \\
f^{(0,0)}\left(\cdot+\epsilon_{2}\right)-f^{(0,0)}(\cdot)-f^{(0,1)}(\cdot)-\frac{1}{2} f^{(0,2)}(\cdot) \\
f^{(1,0)}\left(\cdot+\epsilon_{2}\right)-f^{(1,0)}(\cdot)-f^{(1,1)}(\cdot) \\
f^{(0,1)}\left(\cdot+\epsilon_{2}\right)-f^{(0,1)}(\cdot)-f^{(0,2)}(\cdot) \\
f^{(2,0)}(\cdot) \\
f^{(1,1)}(\cdot) \\
f^{(0,2)}(\cdot)
\end{array}\right] \in \mathbb{R}^{12} .
$$

As in one variable we define the complete (partial and total) Taylor operators as

$$
\widetilde{T}_{d}^{j}:=\left[\begin{array}{ccccc}
\Delta_{j} \boldsymbol{I}_{0} & \boldsymbol{T}_{0,1}^{j} & \cdots & \boldsymbol{T}_{0, d-1}^{j} & \boldsymbol{T}_{0, d}^{j} \\
& \Delta_{j} \boldsymbol{I}_{1} & \ddots & \vdots & \vdots \\
& & \ddots & \boldsymbol{T}_{d-2, d-1}^{j} & \vdots \\
& & & \Delta_{j} \boldsymbol{I}_{d-1} & \boldsymbol{T}_{d-1, d}^{j} \\
& & & & \Delta_{j} \boldsymbol{I}_{d}
\end{array}\right], \quad \widetilde{T}_{d}:=\left[\begin{array}{c}
\widetilde{T}_{d}^{1} \\
\vdots \\
\widetilde{T}_{d}^{s}
\end{array}\right] .
$$

Again for the example $s=2$ and $d=2$, we obtain

$$
\widetilde{T}_{2} \boldsymbol{f}=\left[\begin{array}{c}
f^{(0,0)}\left(\cdot+\epsilon_{1}\right)-f^{(0,0)}(\cdot)-f^{(1,0)}(\cdot)-\frac{1}{2} f^{(2,0)}(\cdot) \\
f^{(1,0)}\left(\cdot+\epsilon_{1}\right)-f^{(1,0)}(\cdot)-f^{(2,0)}(\cdot) \\
f^{(0,1)}\left(\cdot+\epsilon_{1}\right)-f^{(0,1)}(\cdot)-f^{(1,1)}(\cdot) \\
f^{(2,0)}\left(\cdot+\epsilon_{1}\right)-f^{(2,0)}(\cdot) \\
f^{(1,1)}\left(\cdot+\epsilon_{1}\right)-f^{(1,1)}(\cdot) \\
f^{(0,2)}\left(\cdot+\epsilon_{1}\right)-f^{(0,2)}(\cdot) \\
f^{(0,0)}\left(\cdot+\epsilon_{2}\right)-f^{(0,0)}(\cdot)-f^{(0,1)}(\cdot)-\frac{1}{2} f^{(0,2)}(\cdot) \\
f^{(1,0)}\left(\cdot+\epsilon_{2}\right)-f^{(1,0)}(\cdot)-f^{(1,1)}(\cdot) \\
f^{(0,1)}\left(\cdot+\epsilon_{2}\right)-f^{(0,1)}(\cdot)-f^{(0,2)}(\cdot) \\
f^{(2,0)}\left(\cdot+\epsilon_{2}\right)-f^{(2,0)}(\cdot) \\
f^{(1,1)}\left(\cdot+\epsilon_{2}\right)-f^{(1,1)}(\cdot) \\
f^{(0,2)}\left(\cdot+\epsilon_{2}\right)-f^{(0,2)}(\cdot)
\end{array}\right] \in \mathbb{R}^{12} .
$$

Obviously, in the general case, $T_{d}$ and $\widetilde{T}_{d}$ map an $r_{d}$-vector valued sequence to a $s \cdot r_{d}$-vector valued sequence.

We will denote the unit vectors in $\mathbb{R}^{r_{d}}$ by $\boldsymbol{e}_{\alpha},|\alpha| \leq d$, and the unit vectors in $\mathbb{R}^{s r_{d}}$ by $\boldsymbol{e}_{j, \alpha}, j=1, \ldots, s,|\alpha| \leq d$. 
Definition 10 The mask $\boldsymbol{A} \in \ell^{r_{d} \times r_{d}}\left(\mathbb{Z}^{s}\right)$ is said to satisfy the spectral condition of order $d^{\prime} \leq d$ if there exists a basis of $\Pi_{d^{\prime}}$ consisting of polynomials $p_{\alpha} \in \Pi_{|\alpha|},|\alpha| \leq d^{\prime}$, such that

$$
S_{\boldsymbol{A}} \boldsymbol{v}_{\alpha}=2^{-|\alpha|} \boldsymbol{v}_{\alpha}, \quad \boldsymbol{v}_{\alpha}=\boldsymbol{v}_{p_{\alpha}}
$$

Remark 11 Since $\left\{p_{\beta}\right\}_{|\beta| \leq k}$ is a basis of $\Pi_{k}$, for any $\alpha$ such that $|\alpha|=k$, the monomial $\frac{1}{\alpha !} x^{\alpha}$ can be written as

$$
\frac{1}{\alpha !} x^{\alpha}=\sum_{|\beta|=k} \lambda_{\alpha, \beta} p_{\beta}+\sum_{|\beta|<k} \lambda_{\alpha, \beta} p_{\beta} .
$$

Let $q_{\alpha}(x)=\sum_{|\beta|<k} \lambda_{\alpha, \beta} p_{\beta}$. By (18), the polynomial $m_{\alpha}(x)=\sum_{|\beta|=k} \lambda_{\alpha, \beta} p_{\beta}=$ $\frac{1}{\alpha !} x^{\alpha}-q_{\alpha}(x)$ satisfies $S_{\boldsymbol{A}} \boldsymbol{v}_{m_{\alpha}}=2^{-|\alpha|} \boldsymbol{v}_{m_{\alpha}}$. Replacing $p_{\alpha}$ by $m_{\alpha}$ if necessary, we can thus always assume that the polynomials $p_{\alpha}$ of Definition 10 are normalized in such a way that their leading term is $\frac{1}{\alpha !} x^{\alpha}$.

The counterpart for Lemma 5 is as follows.

Lemma 12 For $p \in \Pi_{d}$ we have that

$$
T_{d} \boldsymbol{v}_{p}=\sum_{j=1}^{s} \sum_{|\alpha|=d} \boldsymbol{e}_{j, \alpha} D^{\alpha} p(0) .
$$

Proof: For $|\alpha|<d$, any $\gamma \in \mathbb{Z}^{s}$ and $j=1, \ldots, s$ there exists some $\xi=\xi_{\alpha, \gamma, j} \in(0,1)$ such that

$$
\boldsymbol{e}_{j, \alpha}^{T} T_{d} \boldsymbol{v}_{p}(\gamma)=\left(T_{d}^{j} \boldsymbol{v}_{p}\right)_{\alpha}(\gamma)=\frac{1}{(d-|\alpha|+1) !} D^{\alpha+(d-|\alpha|+1)} p\left(\gamma+\xi \epsilon_{j}\right)=0
$$

while for $|\alpha|=d$ and $j=1, \ldots, s$

$$
\boldsymbol{e}_{j, \alpha}^{T} T_{d} \boldsymbol{v}_{p}(\gamma)=\alpha ! p_{\alpha}(\gamma)=D^{\alpha} p(0),
$$

which can be combined into

$$
T_{d} \boldsymbol{v}_{p}=\sum_{j=1}^{s} \sum_{|\alpha| \leq d} \boldsymbol{e}_{j, \alpha} \boldsymbol{e}_{j, \alpha}^{T} T_{d} \boldsymbol{v}=\sum_{j=1}^{s} \sum_{|\alpha|=d} \boldsymbol{e}_{j, \alpha} D^{\alpha} p(0),
$$

that is (19).

Corollary $\mathbf{1 3}$ For $p \in \Pi_{d}$ we have that $\widetilde{T}_{d} \boldsymbol{v}_{p}=\mathbf{0}$.

Also the extension of Proposition 7 follows essentially the same lines but now makes use of the associated quotient ideals, cf. [23, 26].

Proposition 14 For some positive integer $N$, let $C \in \ell^{N \times r_{d}}\left(\mathbb{Z}^{s}\right)$. If for $k \leq d$, $S_{\boldsymbol{C}} \boldsymbol{v}_{p}=0$ for all $p \in \Pi_{k}$, then there exists a finitely supported mask $\boldsymbol{B}_{k} \in \ell^{N \times s r_{d}}$ such that

$$
S_{\boldsymbol{C}}=S_{\boldsymbol{B}_{k}} \widetilde{T}_{k}
$$


Proof: Again let us write $C^{*}$ in terms of its column blocks,

$$
\boldsymbol{C}^{*}(z)=\left[\boldsymbol{C}_{0}^{*}(z) \cdots C_{d}^{*}(z)\right], \quad \boldsymbol{C}_{k}^{*}(z)=\left[\boldsymbol{c}_{\alpha}^{*}(z)\right]_{|\alpha|=k}, \quad k=0, \ldots, d .
$$

The proof consists of showing that whenever $S_{\boldsymbol{C}} \boldsymbol{v}_{p}=0$ for all $p \in \Pi_{k}$, then there exist finitely supported masks $\boldsymbol{B}_{j, k} \in \ell^{N \times s_{j}}\left(\mathbb{Z}^{s}\right), j=1, \ldots, s$ and $k=0, \ldots, d$, thus $\boldsymbol{B}_{k} \in \ell^{N \times r_{d}}\left(\mathbb{Z}^{s}\right), k=0, \ldots, d$, such that

$$
\begin{aligned}
& \boldsymbol{C}^{*}(z)= {\left[\boldsymbol{B}_{10}^{*}(z) \cdots \boldsymbol{B}_{1 k}^{*}(z) \boldsymbol{C}_{k+1}^{*}(z) \cdots \boldsymbol{C}_{d}^{*}(z) \mid \cdots\right.} \\
&\left.\cdots \mid \boldsymbol{B}_{s 0}^{*}(z) \cdots \boldsymbol{B}_{s k}^{*}(z) \boldsymbol{C}_{k+1}^{*}(z) \cdots \boldsymbol{C}_{d}^{*}(z)\right]\left[\begin{array}{cc}
\left(\widetilde{T}_{k}^{1}\right)^{*}\left(z^{2}\right) & \mathbf{0} \\
\mathbf{0} & \boldsymbol{I} \\
\vdots & \vdots \\
\left(\widetilde{T}_{k}^{s}\right)^{*}\left(z^{2}\right) & \mathbf{0} \\
\mathbf{0} & \boldsymbol{I}
\end{array}\right] \\
&=: \quad \boldsymbol{B}_{k}^{*}(z) \widetilde{T}_{k}^{*}\left(z^{2}\right), \quad \boldsymbol{I} \in \mathbb{R}^{\left(r_{d}-r_{k}\right) \times\left(r_{d}-r_{k}\right)} .
\end{aligned}
$$

Once more, induction is used to build $\boldsymbol{B}_{k}$ or $\boldsymbol{B}_{k}^{*}(z)$.

For $k=0$ we only have to consider $p=1$, that is $\boldsymbol{v}_{p}=\boldsymbol{e}_{0}$, which leads to $\mathbf{0}=S_{C} e_{0}$ such that, by (4), we have the vector identity

$$
\boldsymbol{c}_{0}^{*}(z)=\sum_{j=1}^{s}\left(z_{j}^{-2}-1\right) \boldsymbol{b}_{0 j}^{*}(z),
$$

and with $\boldsymbol{B}_{0 j}^{*}=\boldsymbol{b}_{0 j}^{*}$ we thus get that

$$
\begin{aligned}
\boldsymbol{C}^{*}(z)= & {\left[\boldsymbol{B}_{01}^{*}(z) \boldsymbol{C}_{1}^{*}(z) \cdots \boldsymbol{C}_{d}^{*}(z)|\cdots| \boldsymbol{B}_{0 s}^{*}(z) \boldsymbol{C}_{1}^{*}(z) \cdots \boldsymbol{C}_{d}^{*}(z)\right]\left[\begin{array}{cc}
z_{1}^{-2}-1 & \mathbf{0} \\
\mathbf{0} & \boldsymbol{I} \\
\vdots & \vdots \\
z_{s}^{-2}-1 & \mathbf{0} \\
\mathbf{0} & \boldsymbol{I}
\end{array}\right] } \\
= & \boldsymbol{B}_{0}^{*}(z)\left[\begin{array}{cc}
\left(\widetilde{T}_{0}^{1}\right)^{*}\left(z^{2}\right) & \mathbf{0} \\
\mathbf{0} & \boldsymbol{I} \\
\vdots & \vdots \\
\left(\widetilde{T}_{0}^{s}\right)^{*}\left(z^{2}\right) & \mathbf{0} \\
\mathbf{0} & \boldsymbol{I}
\end{array}\right]=\boldsymbol{B}_{0}^{*}(z) \widetilde{T}_{0}^{*}\left(z^{2}\right),
\end{aligned}
$$

which is (21) for $k=0$.

Now suppose that the validity of (21) has been proved for some $k$ and assume that $S_{\boldsymbol{C}} \boldsymbol{v}_{p}=0$ for all $p \in \Pi_{k+1}$. By the induction hypothesis, (21) holds, so that $\boldsymbol{C}^{*}(z)=\boldsymbol{B}_{k}^{*}(z) \widetilde{T}_{k}^{*}\left(z^{2}\right)$. Fix $\alpha \in \mathbb{N}_{0}^{s}$ with $|\alpha|=k+1$. For the monic polynomial $p_{\alpha}$ with $p_{\alpha}(x)-\frac{1}{\alpha !} x^{\alpha} \in \Pi_{|\alpha|-1}$, we again consider the truncated vector sequence

$$
\boldsymbol{v}_{p}^{\prime}=\left[\left(\boldsymbol{v}_{p}\right)_{\beta}\right]_{|\beta| \leq k} \in \ell^{r_{k}}\left(\mathbb{Z}^{s}\right)
$$


for which (20) yields that there exist some $\xi \in \mathbb{R}^{d}$ such that for $j=1, \ldots, s$ and $|\beta| \leq k$

$$
\begin{aligned}
\boldsymbol{e}_{j, \beta}^{T} \widetilde{T}_{k} \boldsymbol{v}_{p}^{\prime} & =\left(T_{d}^{j} \boldsymbol{v}_{p}\right)_{\beta}=\frac{1}{(k-|\beta|+1) !} D^{\beta+(k-|\beta|+1) \epsilon_{j}} p(\xi) \\
& =\frac{1}{(k-|\beta|+1) !} \delta_{\beta+(k-|\beta|+1) \epsilon_{j}, \alpha}
\end{aligned}
$$

hence,

$$
\widetilde{T}_{k}^{j} \boldsymbol{v}_{p}^{\prime}=\sum_{\ell=0}^{k} \frac{1}{(k-\ell+1) !} \boldsymbol{e}_{\alpha-(k-\ell+1) \epsilon_{j}}=\sum_{\ell=1}^{k+1} \frac{1}{\ell !} \boldsymbol{e}_{\alpha-\ell \epsilon_{j}}=: \boldsymbol{w}_{j} \in \mathbb{R}^{r_{k}},
$$

with the usual convention that $\boldsymbol{e}_{\beta}=\mathbf{0}$ whenever $\beta \in \mathbb{Z}^{s} \backslash \mathbb{N}_{0}^{s}$, that is, whenever $\beta$ has a negative component. The definition of $\boldsymbol{w}_{j}$ in (22) is also valid in $\mathbb{R}^{r_{d}}$, and we will denote this natural embedding by $\widetilde{\boldsymbol{w}}_{j}$. Now,

$$
\begin{aligned}
\mathbf{0} & =S_{\boldsymbol{C}} \boldsymbol{v}_{p}=S_{\boldsymbol{B}_{k}} \widetilde{T}_{k}\left(\boldsymbol{v}_{p}^{\prime}+\boldsymbol{e}_{\alpha}\right)=S_{\boldsymbol{B}_{k}}\left[\begin{array}{c}
\widetilde{\boldsymbol{w}}_{1}+\boldsymbol{e}_{\alpha} \\
\vdots \\
\widetilde{\boldsymbol{w}}_{s}+\boldsymbol{e}_{\alpha}
\end{array}\right] \\
& =S_{\boldsymbol{B}_{k}} \sum_{j=1}^{s}\left(\boldsymbol{e}_{j, \alpha}+\sum_{\ell=1}^{k+1} \frac{1}{\ell !} \boldsymbol{e}_{j, \alpha-\ell \epsilon_{j}}\right)
\end{aligned}
$$

which can be summarized by stating that

$$
\mathbf{0}=S_{\boldsymbol{B}_{k}} \boldsymbol{w}_{\alpha}, \quad \boldsymbol{w}_{\alpha}:=\sum_{j=1}^{s}\left(\boldsymbol{e}_{j, \alpha}+\sum_{\ell=1}^{k+1} \frac{1}{\ell !} \boldsymbol{e}_{j, \alpha-\ell \epsilon_{j}}\right) \in \mathbb{R}^{s r_{d}}, \quad|\alpha|=k+1 .
$$

Recalling that $\left(\boldsymbol{B}_{k}\right)_{j, \alpha}^{*}=\boldsymbol{c}_{\alpha}^{*}$ for $j=1, \ldots, s$ and $|\alpha|=k+1,(23)$ means in terms of the columns of $\boldsymbol{B}_{k}$ that for $j=1, \ldots, s$ and $|\alpha|=k+1$

$$
0=\boldsymbol{c}_{\alpha}^{*}(z)+\sum_{\ell=1}^{k+1} \frac{1}{\ell !} \boldsymbol{b}_{j, \alpha-\ell \epsilon_{j}}^{*}(z), \quad z \in\{ \pm 1\}^{s},
$$

so that there exist $\boldsymbol{b}_{m, j, \alpha}^{*}, m=1, \ldots, s,|\alpha|=k+1$, such that

$$
\boldsymbol{c}_{\alpha}^{*}(z)+\sum_{\ell=1}^{k+1} \frac{1}{\ell !} \boldsymbol{b}_{j, \alpha-\ell \epsilon_{j}}^{*}(z)=\frac{1}{s} \sum_{m=1}^{s}\left(z_{m}^{-2}-1\right) \boldsymbol{b}_{m, j, \alpha}^{*}(z) .
$$

Averaging this identity over $j$, setting $\boldsymbol{b}_{m, \alpha}^{*}(z):=\frac{1}{s} \sum_{j=1}^{s} \boldsymbol{b}_{m, j, \alpha}^{*}(z)$ and replacing $m$ 
by $j$, we obtain

$$
\begin{aligned}
\boldsymbol{c}_{\alpha}^{*}(z) & =\frac{1}{s} \sum_{j=1}^{s}\left(-\sum_{\ell=1}^{k+1} \frac{1}{\ell !} \boldsymbol{b}_{j, \alpha-\ell \epsilon_{j}}^{*}(z)+\left(z_{j}^{-2}-1\right) \boldsymbol{b}_{j, \alpha}^{*}(z)\right) \\
& =\frac{1}{s} \sum_{j=1}^{s}\left[\boldsymbol{B}_{j, 0}^{*}(z) \cdots \boldsymbol{B}_{j, k+1}^{*}(z)\right]\left[\begin{array}{c}
-\boldsymbol{w}_{j} \\
\left(z_{j}^{-2}-1\right) \boldsymbol{e}_{\alpha}
\end{array}\right] \\
& =\frac{1}{s}\left[\boldsymbol{B}_{j, 0}^{*}(z) \ldots \boldsymbol{B}_{j, k+1}^{*}(z)\right]_{j=1, \ldots, s}\left[\begin{array}{c}
\left(z_{1}^{-2}-1\right) \boldsymbol{e}_{\alpha} \\
\vdots \\
-\boldsymbol{w}_{s} \\
\left(z_{s}^{-2}-1\right) \boldsymbol{e}_{\alpha}
\end{array}\right],
\end{aligned}
$$

which can be combined into

$$
\boldsymbol{C}_{k+1}^{*}(z)=\frac{1}{s}\left[\boldsymbol{B}_{j, 0}^{*}(z) \cdots \boldsymbol{B}_{j, k+1}^{*}(z)\right]_{j=1, \ldots, s}\left[\begin{array}{c}
-\boldsymbol{w}_{1} \mathbf{1}_{s_{k+1}}^{T} \\
\left(z_{1}^{-2}-1\right) \boldsymbol{I}_{s_{k+1}} \\
\vdots \\
-\boldsymbol{w}_{s} \mathbf{1}_{s_{k+1}}^{T} \\
\left(z_{s}^{-2}-1\right) \boldsymbol{I}_{s_{k+1}}
\end{array}\right] .
$$

If we then substitute this expression for the column $C_{k+1}^{*}$ of

$$
\boldsymbol{B}_{k}^{*}(z)=\left[\boldsymbol{B}_{j, 0}^{*}(z) \cdots \boldsymbol{B}_{j, k}^{*}(z) \boldsymbol{C}_{k+1}^{*}(z) \cdots \boldsymbol{C}_{d}^{*}(z)\right]_{j=1, \ldots, s},
$$

we obtain the recursive relationship $\boldsymbol{B}_{k}^{*}(z)=\boldsymbol{B}_{k+1}^{*}(z) \boldsymbol{Y}(z)$ where

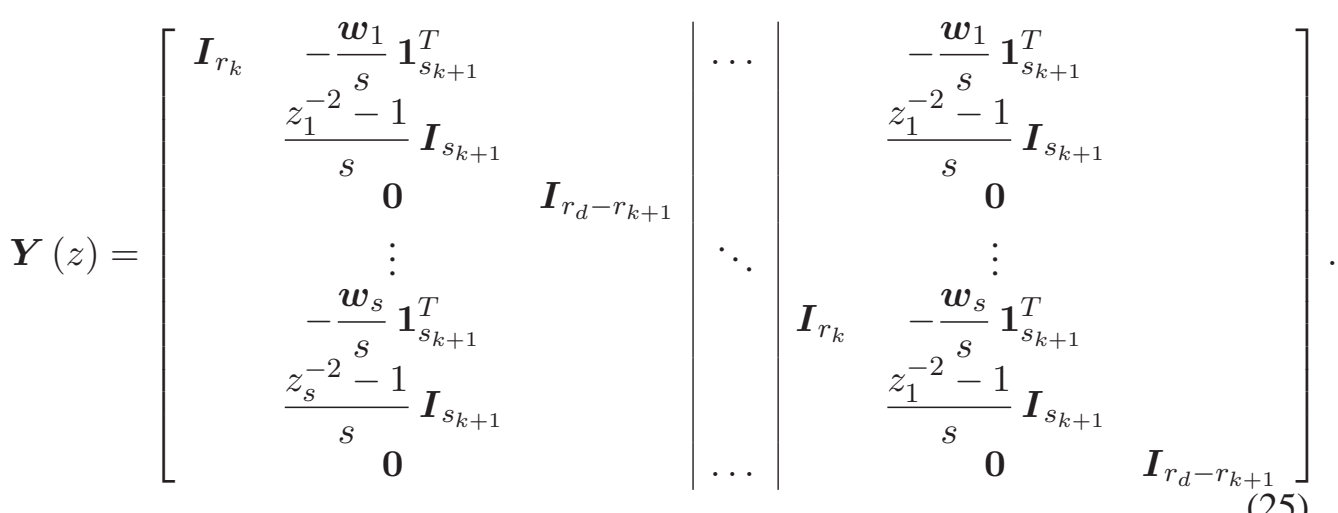

Substituting (25) into (21), we thus get that

$$
\boldsymbol{C}^{*}(z)=\boldsymbol{B}_{k}^{*}(z) T_{k}^{*}\left(z^{2}\right)=\frac{1}{s} \boldsymbol{B}_{k+1}^{*}(z) \boldsymbol{Y}(z) T_{k}^{*}\left(z^{2}\right) .
$$


If we partition $T_{k}^{*}$ according to the structure of $\boldsymbol{Y}$ as

$$
T_{k}^{*}=\left[\begin{array}{ccc}
\left(T_{k}^{1}\right)^{*} & & \\
& \boldsymbol{I}_{s_{k+1}} & \\
& & \boldsymbol{I}_{r_{d}-r_{k+1}} \\
\vdots & \vdots & \vdots \\
\left(T_{k}^{s}\right)^{*} & & \\
& \boldsymbol{I}_{s_{k+1}} & \\
& & \boldsymbol{I}_{r_{d}-r_{k+1}}
\end{array}\right]
$$

then a simple matrix multiplication yields that

$$
\boldsymbol{Y}^{*}(z) T_{k}^{*}\left(z^{2}\right)=\left[\begin{array}{ccc}
\left(T_{k}^{1}\right)^{*}\left(z_{2}\right) & -\boldsymbol{w}_{1} \mathbf{1}^{T} & \mathbf{0} \\
\mathbf{0} & \left(z_{1}^{-2}-1\right) \boldsymbol{I}_{s_{k+1}} & \mathbf{0} \\
\mathbf{0} & \mathbf{0} & \boldsymbol{I} \\
\vdots & \vdots & \vdots \\
\left(T_{k}^{s}\right)^{*}\left(z^{2}\right) & -\boldsymbol{w}_{s} \mathbf{1}^{T} & \mathbf{0} \\
\mathbf{0} & \left(z_{s}^{-2}-1\right) \boldsymbol{I}_{s_{k+1}} & \mathbf{0} \\
\mathbf{0} & \mathbf{0} & \boldsymbol{I}
\end{array}\right]=T_{k+1}^{*}\left(z^{2}\right)
$$

which shows that indeed $\boldsymbol{C}^{*}(z)=\boldsymbol{B}_{k+1}^{*}(z) T_{k+1}^{*}\left(z^{2}\right)$, thus advancing the induction hypothesis and completing the proof.

With an appropriate renormalization like in the proof of Theorem 4, we thus obtain its counterpart for dimension $s$.

Theorem 15 If the mask $\boldsymbol{A} \in \ell^{r_{d} \times r_{d}}\left(\mathbb{Z}^{s}\right)$ satisfies the spectral condition of order $d$ then there exists two (finitely supported) masks $\boldsymbol{B}, \widetilde{\boldsymbol{B}} \in \ell^{s r_{d} \times s r_{d}}\left(\mathbb{Z}^{s}\right)$ such that

$$
T_{d} S_{\boldsymbol{A}}=2^{-d} S_{\boldsymbol{B}} T_{d}, \quad \text { and } \quad \widetilde{T}_{d} S_{\boldsymbol{A}}=2^{-d} S_{\widetilde{B}} \widetilde{T}_{d} .
$$

Remark 16 It is worthwhile to note that the proof of Theorem 15 gives a constructive method to compute the symbols of the Taylor schemes $\boldsymbol{B}$ and $\widetilde{\boldsymbol{B}}$. The crucial step is the computation of the decomposition in (24), a task that is already implemented in some Computer Algebra Systems, for example in CoCoA, [2]. Recall, however, that due to the existence of syzygies this decomposition is usually not unique, cf. [3] and so also $\boldsymbol{B}$ and $\widetilde{\boldsymbol{B}}$ are not unique.

Definition 17 With the hypotheses of the previous Theorem, the subdivision schemes $S_{B}$ is called the Taylor subdivision scheme associated with $H_{A}$ and the scheme $S_{\widetilde{B}}$ is the complete Taylor subdivision scheme.

Remark 18 For initial data $\boldsymbol{f}_{0} \in \ell^{r_{d}}\left(\mathbb{Z}^{s}\right)$, we consider the sequence of iterations $f_{n}, n \in \mathbb{N}$ built from the scheme $H_{A}$ defined in (6). If we now define the associated sequence

$$
\boldsymbol{g}_{n}:=2^{n d} T_{d} \boldsymbol{D}^{n} \boldsymbol{f}_{n} \in \ell^{s r_{d}}\left(\mathbb{Z}^{s}\right), \quad n \in \mathbb{N},
$$


and if $\boldsymbol{A}$ satisfies the spectral condition, then we deduce from (6) that for $n \in \mathbb{N}$ we have

$$
\begin{aligned}
\boldsymbol{g}_{n} & =2^{n d} T_{d} \boldsymbol{D}^{n} \boldsymbol{f}_{n}=2^{n d} T_{d} S_{\boldsymbol{A}} \boldsymbol{D}^{n-1} \boldsymbol{f}_{n-1}=2^{(n-1) d} S_{\boldsymbol{B}} T_{d} \boldsymbol{D}^{n-1} \boldsymbol{f}_{n-1} \\
& =S_{\boldsymbol{B}} \boldsymbol{g}_{n-1}=\left(S_{\boldsymbol{B}}\right)^{n} \boldsymbol{g}_{0},
\end{aligned}
$$

hence $\boldsymbol{g}_{n}$ is generated by the stationary subdivision scheme $S_{B}$.

Similarly if $\boldsymbol{h}_{n}:=2^{n d} \widetilde{T}_{d} \boldsymbol{D}^{n} \boldsymbol{f}_{n}$ then, $\boldsymbol{h}_{n}=\left(S_{\widetilde{\boldsymbol{B}}}\right)^{n} \boldsymbol{h}_{0}$.

\section{Convergence of Subdivision Operators}

Definition 19 Let $\boldsymbol{B} \in \ell^{r \times r}\left(\mathbb{Z}^{s}\right)$ be a mask and $S_{\boldsymbol{B}}: \ell^{r}\left(\mathbb{Z}^{s}\right) \rightarrow \ell^{r}\left(\mathbb{Z}^{s}\right)$ the associated stationary subdivision operator defined in (1). The operator is $C^{0}$-convergent if for any data $\boldsymbol{g}_{0} \in \ell^{r}\left(\mathbb{Z}^{s}\right)$ and corresponding sequence of refinements $\boldsymbol{g}_{n}=S_{B}^{n} \boldsymbol{g}_{0}$ there exists a function $\Psi_{\boldsymbol{g}} \in C\left(\mathbb{R}^{s}, \mathbb{R}^{r}\right)$ such that for any compact $K \subset \mathbb{R}^{s}$ there exists a sequence $\varepsilon_{n}$ with limit 0 that satisfies

$$
\max _{\alpha \in \mathbb{Z}^{s} \cap 2^{n} K}\left\|\boldsymbol{g}_{n}(\alpha)-\Psi_{\boldsymbol{g}}\left(2^{-n} \alpha\right)\right\|_{\infty} \leq \varepsilon_{n}
$$

Definition 20 Let $\boldsymbol{A} \in \ell^{r_{d} \times r_{d}}\left(\mathbb{Z}^{s}\right)$ be a mask and $H_{\boldsymbol{A}}$ the associated Hermite subdivision scheme on $\ell^{r_{d}}\left(\mathbb{Z}^{s}\right)$ as defined in (6). The scheme is convergent if for any data $\boldsymbol{f}_{0} \in \ell^{r_{d}}\left(\mathbb{Z}^{s}\right)$ and the corresponding sequence of refinements $\boldsymbol{f}_{n}$, there exists a function $\Phi=\left[\phi_{\alpha}\right]_{|\alpha| \leq d} \in C\left(\mathbb{R}^{s}, \mathbb{R}^{r_{d}}\right)$ such that for any compact $K \subset \mathbb{R}^{s}$ there exists a sequence $\varepsilon_{n}$ with limit 0 which satisfies

$$
\max _{|\alpha| \leq d} \max _{\beta \in \mathbb{Z}^{s} \cap 2^{n} K}\left|f_{n}^{(\alpha)}(\beta)-\phi_{\alpha}\left(2^{-n} \beta\right)\right| \leq \varepsilon_{n} .
$$

The scheme $H_{\boldsymbol{A}}$ is said to be $C^{d}$-convergent if moreover $\phi_{0} \in C^{d}\left(\mathbb{R}^{s}, \mathbb{R}\right)$ and

$$
\frac{\partial^{\alpha} \phi_{0}}{\partial x^{\alpha}}=\phi_{\alpha}, \quad|\alpha| \leq d
$$

Theorem 21 Given a mask $\boldsymbol{A} \in \ell^{r_{d}}\left(\mathbb{Z}^{s}\right)$ which satisfies the spectral condition. Suppose that for any data $\boldsymbol{f}_{0} \in \ell^{r_{d}}\left(\mathbb{Z}^{s}\right)$ and associated refinement sequence $\boldsymbol{f}_{n}$ of the Hermite scheme $H_{A}$,

1. the sequence $\boldsymbol{f}_{n}(0)$ converges to a limit $\boldsymbol{y} \in \mathbb{R}^{r_{d}}$,

2. the associated Taylor subdivision scheme $S_{B}$ is $C^{0}$-convergent and for any initial data $\boldsymbol{g}_{0}=T_{d} \boldsymbol{f}_{0}$, the limit function $\Psi=\Psi_{\boldsymbol{g}} \in C\left(\mathbb{R}^{s}, \mathbb{R}^{s r_{d}}\right)$ satisfies

$$
\Psi=\mathbf{1}_{s} \otimes\left[\begin{array}{c}
\mathbf{0}_{r_{d}-s_{d}} \\
\Psi_{d}
\end{array}\right]=\left[\begin{array}{c}
\mathbf{0} \\
\Psi_{d} \\
\vdots \\
\mathbf{0} \\
\Psi_{d}
\end{array}\right], \quad \Psi_{d}=\left[\psi_{\alpha}\right]_{|\alpha|=d} .
$$

Then $H_{A}$ is convergent. 
The construction of the limit function $\Phi$ of $H_{\boldsymbol{A}}$ starts with a lemma in the special case $d=1$.

Lemma 22 Given a sequence of refinements

$$
\boldsymbol{h}_{n}=\left[\begin{array}{l}
h_{n}^{(0)} \\
\boldsymbol{h}_{n}^{(1)}
\end{array}\right] \in \ell\left(\mathbb{Z}^{s}, \mathbb{R}^{s+1}\right), \quad \boldsymbol{h}_{n}^{(1)}=\left[h_{n}^{\epsilon_{j}}\right]_{j=1, \ldots, s}
$$

such that

1. there exists a constant $c$ in $\mathbb{R}$ such that $\lim _{n \rightarrow+\infty} h_{n}^{(0)}(0)=c$,

2. there exists a function $\Xi \in C\left(\mathbb{R}^{s}, \mathbb{R}^{s}\right)$ such that for any compact subset $K$ of $\mathbb{R}^{s}$ there exists a sequence $\mu_{n}$ with limit 0 and

$$
\begin{aligned}
\max _{\alpha \in 2^{n} K \cap \mathbb{Z}^{s}}\left\|\boldsymbol{h}_{n}^{(1)}(\alpha)-\Xi\left(2^{-n} \alpha\right)\right\|_{\infty} \leq \mu_{n}, \\
\max _{\alpha \in 2^{n} K \cap \mathbb{Z}^{s}}\left\|2^{n} \Delta h_{n}^{(0)}(\alpha)-\boldsymbol{h}_{n}^{(1)}(\alpha)\right\|_{\infty} \leq \mu_{n} .
\end{aligned}
$$

Then there exists for any compact $K$ a sequence $\theta_{n}$ with limit 0 such that the function

$$
\varphi(x)=c+\int_{0}^{1} x^{T} \Xi(t x) d t, \quad x \in \mathbb{R}^{s},
$$

satisfies

$$
\max _{\alpha \in 2^{n} K \cap \mathbb{Z}^{s}}\left\|h_{n}^{(0)}(\alpha)-\varphi\left(2^{-n} \alpha\right)\right\| \leq \theta_{n},
$$

Before we prove this lemma, let us recall some useful notations and concepts. For $f \in C\left(K, \mathbb{R}^{k}\right)$ we will write the modulus of continuity as

$$
\omega(f, h)=\max \left\{\|f(x)-f(y)\|_{\infty}: x, y \in K,\|x-y\|_{1} \leq h\right\} .
$$

Since $K$ is compact, $f$ is uniformly continuous and this value tends to 0 when $h$ tends to 0 .

Given a multiindex $\tau \in \mathbb{Z}^{s}$, a path from 0 to $\tau$ is a sequence $\tau^{j}, j=0, \ldots, n$, such that $\tau^{0}=0, \tau^{n}=\tau$ and $\tau^{j+1}-\tau^{j} \in\left\{ \pm \epsilon_{k}\right\}_{k=1, \ldots, s}$. In other words, a path goes from 0 to $\tau$ in steps of unit vectors. A path is called direct if it has minimal length, i.e., if $n=\|\tau\|_{1}=|\tau|$. Clearly, to any $\tau$ there exists at least one direct path.

Proof: Clearly, the function $\varphi$ from (33) is continuous.

We prove (34) for $K=[-L, L]^{s}$ since any compact subset of $\mathbb{R}^{s}$ is included in a such hypercube.

Let $n$ be an even integer and let us fix $\alpha \in 2^{n} K \cap \mathbb{Z}^{s}$, which we decompose into $\alpha=\beta+\tau$ where

$$
\beta=2^{n / 2} \beta^{\prime}, \quad \beta^{\prime} \in 2^{n / 2} K \cap \mathbb{Z}^{s}, \quad\|\alpha-\beta\|_{\infty} \leq 2^{n / 2} .
$$

Such a $\beta^{\prime}$ can be obtained by rounding $2^{-n / 2} \alpha$ componentwise to the next integer, yielding $\left\|2^{-n / 2} \alpha-\beta^{\prime}\right\|_{\infty}<1$, hence $\|\alpha-\beta\|_{\infty}<2^{n / 2}$. (see figure). 


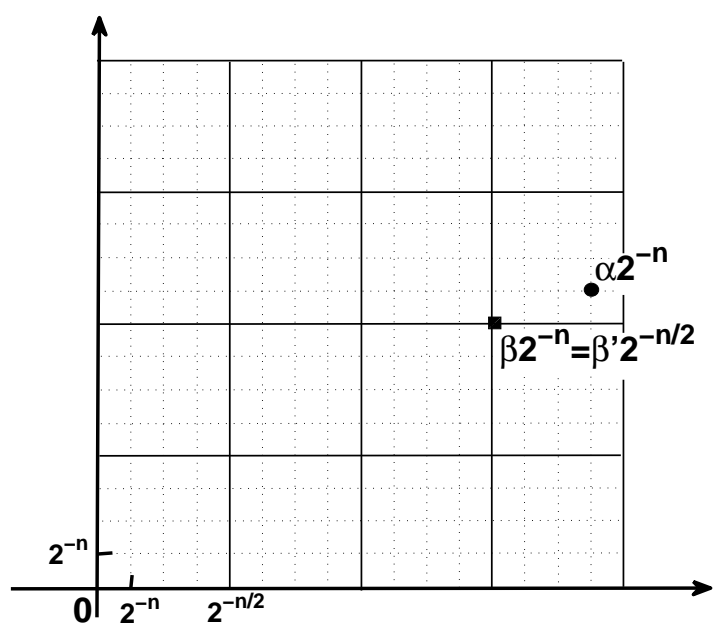

The grids with $2^{-n}$ and $2^{-n / 2}$

Then

$$
\begin{aligned}
& \left|h_{n}^{(0)}(\alpha)-\varphi\left(2^{-n} \alpha\right)\right| \\
& \quad \leq\left|h_{n}^{(0)}(\alpha)-h_{n}^{(0)}(\beta)\right|+\left|h_{n}^{(0)}(\beta)-\varphi\left(2^{-n} \beta\right)\right|+\left|\varphi\left(2^{-n} \beta\right)-\varphi\left(2^{-n} \alpha\right)\right|
\end{aligned}
$$

and we will bound each of these terms separately.

Majorization of $\left|h_{n}^{(0)}(\alpha)-h_{n}^{(0)}(\beta)\right|:$ We choose a direct path $\left\{\tau^{j}\right\}_{j=0, \ldots,|\tau|}$ from 0 to $\tau$ and deduce from (32) that there exists $k \in\{1, \ldots, s\}$ such that

$$
\left|h_{n}^{(0)}\left(\beta+\tau^{j+1}\right)-h_{n}^{(0)}\left(\beta+\tau^{j}\right)\right| \leq 2^{-n}\left(\mu_{n}+\left|h_{n}^{\epsilon_{k}}(\beta+\eta)\right|\right)
$$

where $\eta$ is either $\tau^{j}$ or $\tau^{j+1}$, depending on the sign of $\tau^{j+1}-\tau^{j}$. Involving also (31), we then obtain

$$
\begin{aligned}
\left|h_{n}^{(0)}\left(\beta+\tau^{j+1}\right)-h_{n}^{(0)}\left(\beta+\tau^{j}\right)\right| & \leq 2^{-n}\left(2 \mu_{n}+\left|\xi_{k}(\beta+\eta)\right|\right) \\
& \leq 2^{-n}\left(2 \mu_{n}+\|\Xi\|_{\infty}\right)
\end{aligned}
$$

where the right hand side is independent of $j$. Hence,

$$
\begin{aligned}
\left|h_{n}^{(0)}(\alpha)-h_{n}^{(0)}(\beta)\right| & =\sum_{j=0}^{|\tau|-1}\left|h_{n}^{(0)}\left(\beta+\tau^{j+1}\right)-h_{n}^{(0)}\left(\beta+\tau^{j}\right)\right| \\
& \leq 2^{-n} \sum_{j=0}^{|\tau|-1}\left(2 \mu_{n}+\|\Xi\|_{\infty}\right)=2^{-n}|\tau|\left(2 \mu_{n}+\|\Xi\|_{\infty}\right)
\end{aligned}
$$

and since $|\tau| \leq s 2^{n / 2}$, we obtain the desired bound

$$
\left|h_{n}^{(0)}(\alpha)-h_{n}^{(0)}(\beta)\right| \leq 2^{-n / 2} s\left(2 \mu_{n}+\|\Xi\|_{\infty}\right) .
$$


Majorization of $\left|h_{n}^{(0)}(\beta)-\varphi\left(2^{-n} \beta\right)\right|:$ Since $\beta=2^{n / 2} \beta^{\prime}$ with $\beta^{\prime} \in \mathbb{Z}^{s}$,

$$
\begin{aligned}
h_{n}^{(0)}(\beta) & =h_{n}^{(0)}(0)+\sum_{j=0}^{2^{n / 2}-1} h_{n}^{(0)}\left((j+1) \beta^{\prime}\right)-h_{n}^{(0)}\left(j \beta^{\prime}\right) \\
\varphi\left(2^{-n} \beta\right) & =c+\sum_{j=0}^{2^{n / 2}-1} 2^{-n} \int_{j 2^{-n / 2}}^{(j+1) 2^{-n / 2}} \beta^{T} \Xi\left(2^{-n} t \beta\right) d t,
\end{aligned}
$$

and therefore

$$
\begin{aligned}
& \left|h_{n}^{(0)}(\beta)-\varphi\left(2^{-n} \beta\right)\right| \\
& \leq\left|h_{n}^{(0)}(0)-c\right|+\sum_{j=0}^{2^{n / 2}-1}\left|h_{n}^{(0)}\left((j+1) \beta^{\prime}\right)-h_{n}^{(0)}\left(j \beta^{\prime}\right)-2^{-n} \beta^{T} \Xi\left(2^{-n} j \beta^{\prime}\right)\right| \\
& \quad+2^{-n} \sum_{j=0}^{2^{n / 2}-1}\left|\beta^{T} \Xi\left(j \beta^{\prime} / 2^{n}\right)-\int_{j 2^{-n / 2}}^{(j+1) 2^{-n / 2}} \beta^{T} \Xi\left(2^{-n} t \beta\right) d t\right|
\end{aligned}
$$

The first term in the right hand side sum converges to zero by assumption and we can assume that the sequence $\mu_{n}$ is chosen in such a way that $\left|h_{n}^{(0)}(0)-c\right| \leq \mu_{n}$, for the second one we let $\left(\tau^{k}\right)_{k=0, \ldots,\left|\beta^{\prime}\right|}$ denote a direct path from 0 to $\beta^{\prime}$, hence

$$
\beta^{\prime}=\sum_{k=0}^{\left|\beta^{\prime}\right|-1} \tau^{k+1}-\tau^{k} .
$$

Since $2^{-n}|\beta| \leq s L$ and since $\tau^{k}$ is a path from 0 to $\beta^{\prime}$ we have that

$$
2^{-n}\left|\tau^{k}\right| \leq 2^{-n}\left|\beta^{\prime}\right|=2^{-n} 2^{-n / 2}|\beta| \leq s L 2^{-n / 2} .
$$

For any $\gamma \in \mathbb{Z}^{s}$ and $\ell$ such that $\tau^{k+1}-\tau^{k}= \pm \epsilon_{\ell}$, we observe that

$$
\begin{aligned}
\mid h_{n}^{(0)} & \left(\gamma+\tau^{k+1}\right)-h_{n}^{(0)}\left(\gamma+\tau^{k}\right) \mp 2^{-n} \xi_{\ell}\left(2^{-n} \gamma\right) \mid \\
\leq & \left|h_{n}^{(0)}\left(\gamma+\tau^{k+1}\right)-h_{n}^{(0)}\left(\gamma+\tau^{k}\right) \mp 2^{-n} h_{n}^{\epsilon_{\ell}}\left(\gamma+\tau^{k}\right)\right| \\
& +2^{-n}\left|h_{n}^{\epsilon_{\ell}}\left(\gamma+\tau^{k}\right)-\xi_{\ell}\left(2^{-n} \gamma\right)\right| \\
\leq & 2^{-n}\left(\mu_{n}+\left|h_{n}^{\epsilon_{\ell}}\left(\gamma+\tau^{k}\right)-\xi_{\ell}\left(\frac{\gamma+\tau^{k}}{2^{n}}\right)\right|\right) \\
& \quad+2^{-n}\left|\xi_{\ell}\left(\frac{\gamma+\tau^{k}}{2^{n}}\right)-\xi_{\ell}\left(2^{-n} \gamma\right)\right| \\
& \leq 2^{-n}\left(2 \mu_{n}+s L \omega\left(\Xi, 2^{-n / 2}\right)\right) \leq 2^{-n}\left(2 \mu_{n}+s L \omega\left(\Xi, 2^{-n / 2}\right)\right),
\end{aligned}
$$

which can be rewritten as

$$
\begin{aligned}
& \left|h_{n}^{(0)}\left(\gamma+\tau^{k+1}\right)-h_{n}^{(0)}\left(\gamma+\tau^{k}\right)-2^{-n}\left(\tau^{k+1}-\tau^{k}\right)^{T} \Xi\left(2^{-n} \gamma\right)\right| \\
& \quad \leq 2^{-n}\left(2 \mu_{n}+s L \omega\left(\Xi, 2^{-n / 2}\right)\right) .
\end{aligned}
$$


again with a right hand side independent of $\gamma$ and $k$. Consequently, for $\gamma \in \mathbb{Z}^{s}$,

$$
\begin{aligned}
& \left|h_{n}^{(0)}\left(\gamma+\beta^{\prime}\right)-h_{n}^{(0)}(\gamma)-2^{-n} \beta^{\prime T} \Xi\left(2^{-n} \gamma\right)\right| \\
& \quad \leq \sum_{k=0}^{\left|\beta^{\prime}\right|-1}\left|h_{n}^{(0)}\left(\gamma+\tau^{k+1}\right)-h_{n}^{(0)}\left(\gamma+\tau^{k}\right)-2^{-n}\left(\tau^{k+1}-\tau^{k}\right) \xi_{\ell(k)}\left(2^{-n} \gamma\right)\right| \\
& \quad \leq\left|\beta^{\prime}\right| 2^{-n}\left(2 \mu_{n}+s L \omega\left(\Xi, 2^{-n / 2}\right)\right) \leq 2^{-n / 2} s L\left(2 \mu_{n}+s L \omega\left(\Xi, 2^{-n / 2}\right)\right),
\end{aligned}
$$

which can be summarized as

$$
\begin{aligned}
& \left|h_{n}^{(0)}\left((j+1) \beta^{\prime}\right)-h_{n}^{(0)}\left(j \beta^{\prime}\right)-2^{-n}{\beta^{\prime}}^{T} \Xi\left(2^{-n} j \beta^{\prime}\right)\right| \\
& \quad \leq 2^{-n / 2} s L\left(2 \mu_{n}+s L \omega\left(\Xi, 2^{-n / 2}\right)\right) .
\end{aligned}
$$

To complete this part of the proof, we have to find an upper bound for

$$
\begin{aligned}
& \left|\beta^{T} \Xi\left(j \beta^{\prime} / 2^{n}\right)-\int_{j 2^{-n / 2}}^{(j+1) 2^{-n / 2}} \beta^{T} \Xi\left(2^{-n} t \beta\right) d t\right| \\
& \quad=\left|\int_{j 2^{-n / 2}}^{(j+1) 2^{-n / 2}} \beta^{T} \Xi\left(2^{-n} t \beta\right) d t-2^{n / 2}{\beta^{\prime}}^{T} \Xi\left(2^{-n} j \beta^{\prime}\right)\right| .
\end{aligned}
$$

Since $\beta=2^{n / 2} \beta^{\prime}$, hence $j \beta^{\prime}=j 2^{-n / 2} \beta$, we get for $t \in\left[j 2^{-n / 2},(j+1) 2^{-n / 2}\right]$ that

$$
\left\|2^{-n} t \beta-2^{-n} j \beta^{\prime}\right\|_{1}=2^{-n}\left\|t \beta-j 2^{-n / 2} \beta\right\|_{1} \leq 2^{-n}|\beta| 2^{-n / 2} \leq s L 2^{-n / 2}
$$

so that $\left\|\Xi\left(2^{-n} t \beta\right)-\Xi\left(2^{-n} j \beta^{\prime}\right)\right\|_{1} \leq s L \omega\left(\Xi, 2^{-n / 2}\right)$. Thus, we deduce that

$$
\begin{aligned}
& \left|\int_{j 2^{-n / 2}}^{(j+1) 2^{-n / 2}} \beta^{T} \Xi\left(2^{-n} t \beta\right) d t-2^{n / 2} \beta^{T} \Xi\left(2^{-n} j \beta^{\prime}\right)\right| \\
& \quad=\left|\beta^{T}\left(\int_{j 2^{-n / 2}}^{(j+1) 2^{-n / 2}} \Xi\left(2^{-n} t \beta\right) d t-\Xi\left(2^{-n} j \beta^{\prime}\right)\right)\right| \\
& \quad \leq|\beta| s L 2^{-n / 2} \omega\left(\Xi, 2^{-n / 2}\right) .
\end{aligned}
$$

Since $2^{-n}|\beta| \leq s L$, we finally get

$$
\begin{aligned}
& \left|2^{-n} \int_{j 2^{-n / 2}}^{(j+1) 2^{-n / 2}} \beta^{T} \Xi\left(2^{-n} t \beta\right) d t-2^{-n} \beta^{T} \Xi\left(2^{-n} j \beta^{\prime}\right)\right| \\
& \quad \leq 2^{-n / 2}(s L)^{2} \omega\left(\Xi, 2^{-n / 2}\right) .
\end{aligned}
$$

We can now substitute the bounds obtained in (40) and (41) into (39) to find that also the second term in (35) is suitably bounded as

$$
\left|h_{n}^{(0)}(\beta)-\varphi\left(2^{-n} \beta\right)\right| \leq(2 s L+1) \mu_{n}+2(s L)^{2} \omega\left(\Xi, 2^{-n / 2}\right) .
$$


Majorization of $\left|\varphi\left(2^{-n} \beta\right)-\varphi\left(2^{-n} \alpha\right)\right|$ : Since $2^{-n}\|\beta-\alpha\|_{1} \leq s 2^{-n / 2}$, we immediately have that

$$
\left|\varphi\left(2^{-n} \beta\right)-\varphi\left(2^{-n} \alpha\right)\right| \leq s \omega\left(\varphi, 2^{-n / 2}\right) .
$$

Final estimate: To finish the proof for even $n$, we just substitute (36), (42) and (43) into (35) yielding

$$
\left|h_{n}^{(0)}(\alpha)-\varphi\left(2^{-n} \alpha\right)\right| \leq \theta_{n}, \quad \alpha \in 2^{n} K \cap \mathbb{Z}^{s} .
$$

with the explicit bound

$$
\theta_{n}=2^{-n / 2}\|\Xi\|_{\infty}+\left(2^{-n / 2} s+2 s L+1\right) \mu_{n}+\left(s+2(s L)^{2}\right) \omega\left(\varphi, 2^{-n / 2}\right),
$$

which tends to 0 for $n \rightarrow \infty$.

If, on the other hand, $n$ is odd, then we build an intermediate coarser grid with stepwidth $2^{(n-1) / 2}$ and associate $\alpha \in 2^{n} K \cap \mathbb{Z}^{s}$ an approximation $\beta \in 2^{(n-1) / 2} K \cap \mathbb{Z}^{s}$, to obtain an analogous result.

Proof of Theorem 21: For initial data $\boldsymbol{f}_{0} \in \ell^{r_{d}}\left(\mathbb{Z}^{s}\right)$ and $\boldsymbol{g}_{0}=T_{d} \boldsymbol{f}_{0}$, the associated Taylor subdivision scheme $S_{B}$ is, by assumption, convergent and the limit function $\Psi_{\boldsymbol{g}} \in C\left(\mathbb{R}^{s}, \mathbb{R}^{s r_{d}}\right)$ satisfies (30). The first hypothesis yields that for any $\alpha$ with $|\alpha|<$ $d$ the sequence $f_{n}^{\alpha}(0)$ converges to $y_{\alpha}$. Then we define $\phi$ recursively as $\phi_{\alpha}=\psi_{\alpha}$, $|\alpha|=d$ and for $|\alpha|=d-1, \ldots, 0$, as

$$
\phi_{\alpha}(x)=c_{\alpha}+\int_{0}^{1} x^{T} \Upsilon_{\alpha}(t x) d t, \quad \Upsilon_{\alpha}=\left[\phi_{\alpha+\epsilon_{j}}\right]_{j=1, \ldots, s} .
$$

Clearly, $\Phi=\left[\phi_{\alpha}\right]_{|\alpha| \leq d}$ is continuous.

Fixing a compact $K \subset \mathbb{R}^{s}$, we will prove by a backward finite recursion for $k=$ $d, d-1, \ldots, 0$ that

$$
\left|f_{n}^{\alpha}(\gamma)-\phi_{\alpha}\left(2^{-n} \gamma\right)\right| \leq \varepsilon_{n}, \quad \gamma \in \mathbb{Z}^{s} \cap 2^{n} K, \quad|\alpha|=k .
$$

The case $k=d$ is an immediate consequence of the convergence of $\boldsymbol{g}_{n}=2^{n d} T_{d} \boldsymbol{D}^{n} \boldsymbol{f}_{n}$, which yields for any $\gamma \in \mathbb{Z}^{s} \cap 2^{n} K$ that

$$
\left|f_{n}^{\alpha}(\gamma)-\psi_{\alpha}\left(2^{-n} \gamma\right)\right| \leq \varepsilon_{n}, \quad|\alpha|=d
$$

while, for $k<d$, and $\alpha \in \mathbb{Z}^{s}$ with $|\alpha|=k$, the convergence of the appropriate component of $\boldsymbol{g}_{n}$ to zero implies that

$$
2^{n(d-|\alpha|)}\left|f_{n}^{\alpha}\left(\gamma+\epsilon_{j}\right)-\sum_{\ell=0}^{d-|\alpha|} \frac{1}{2^{n \ell} \ell !} f_{n}^{\alpha+\ell \epsilon_{j}}(\gamma)\right| \leq \varepsilon_{n}, \quad j=1, \ldots, s,
$$

for a sequence $\varepsilon_{n}$ that tends to zero for $n \rightarrow \infty$.

To prove (45) for $k=d-1$, we define for $\alpha \in \mathbb{Z}^{s}$ with $|\alpha|=d-1$ and $j=$ $1, \ldots, s$, the sequences $h_{n}^{\epsilon_{j}}=f_{n}^{\alpha+\epsilon_{j}}$ as well as $\Xi=\left[\psi_{\alpha+\epsilon_{j}}\right]_{j=1, \ldots, s}$ and $h_{n}^{(0)}=f_{n}^{\alpha}$. Because of (46) and (47), we can apply Lemma 22 and obtain that

$$
\left|f_{n}^{\alpha}(\gamma)-\phi_{\alpha}\left(2^{-n} \gamma\right)\right| \leq \theta_{n}, \quad \gamma \in 2^{n} K \cap \mathbb{Z}^{s}, \quad|\alpha|=d-1,
$$


which is (45) for $k=d-1$.

To prove the recursive step $k \rightarrow k-1,1 \leq k<d$, we observe that for any $\beta \in \mathbb{Z}^{s}$ with $|\beta|=k-1$ and for $j=1, \ldots, s$, we get from (47) that, for $\gamma \in \mathbb{Z}^{s} \cap 2^{n} K$,

$$
\left|2^{n}\left(f_{n}^{\beta}\left(\gamma+\epsilon_{j}\right)-f_{n}^{\beta}(\gamma)\right)-f_{n}^{\beta+\epsilon_{j}}(\gamma)\right| \leq \frac{\varepsilon_{n}}{2^{n(d-k)}}+\sum_{\ell=2}^{d-k} \frac{1}{2^{n(\ell-1)} \ell !}\left|f_{n}^{\beta+\ell \epsilon_{j}}(\gamma)\right| .
$$

Since (45) holds for $|\alpha| \geq k$, it follows for $\ell=2, \ldots,|\beta|-d$

$$
\lim _{n \rightarrow \infty}\left|f_{n}^{\beta+\ell \epsilon_{j}}(\gamma)-\phi_{\beta+\ell \epsilon_{j}}\left(2^{-n} \gamma\right)\right|=0
$$

uniformly for $\gamma \in \mathbb{Z}^{s} \cap 2^{n} K$ and since $\phi_{\beta+\ell \epsilon_{j}}$ is bounded on $K$, so is the sequence $\left|f_{n}^{\beta+\ell \epsilon_{j}}(\gamma)\right|$ on $\mathbb{Z}^{s} \cap 2^{n} K$. Thus the right hand side of (48) converges to zero so that (48) immediately yields (45) for $k$ replaced by $k-1$.

Corollary 23 For $s=1$, given a mask $A \in \ell^{d}(\mathbb{Z})$ which satisfies the spectral condition. Suppose that the associated Taylor subdivision scheme $S_{B}$ is $C^{0}$-convergent with the conditions of Theorem 21 then $H_{A}$ is $C^{d}$-convergent.

Proof: For $s=1$, for $\alpha=d-1, \ldots, 0$, recursively, the function $\phi_{\alpha}$ defined in (44) is $C^{1}$ with $\phi_{\alpha}^{\prime}=\phi_{\alpha+1}$ and $\phi_{0}$ is $C^{d}$ with $\frac{d^{\beta} \phi_{0}}{d x^{\alpha}}=\phi_{\beta}$ for $\beta=0, \ldots, d$.

For $s>1$, the difference between convergence of $H_{A}$ and $C^{d}$-convergence of $H_{A}$ depends on whether $\Psi_{d}$ in (30) is a $d$-th derivative of some function or not. In fact, in the simplest case $d=1$ as in (33), the question reduces to when a $d$-vector of functions in $d$ variables is a gradient of some function. A simple characterization of that property is given in the following Lemma 24 which is most likely known but shall be included for the sake of completeness.

Lemma 24 A vector field $\phi=\left[\varphi_{j}\right]_{j=1, \ldots, s}$ is the gradient of a function $f: \mathbb{R}^{s} \rightarrow \mathbb{R}$ if and only if its distributional derivatives satisfy

$$
\frac{\partial \varphi_{j}}{\partial x_{k}}=\frac{\partial \varphi_{k}}{\partial x_{j}}, \quad j, k=1, \ldots, s .
$$

Proof: If $\phi=\nabla f$, then

$$
\frac{\partial \varphi_{j}}{\partial \xi_{k}}=\frac{\partial^{2} f}{\partial \xi_{j} \partial \xi_{k}}=\frac{\partial \varphi_{k}}{\partial \xi_{j}}
$$

yielding (49). Conversely, if $\phi$ satisfies (49), we set

$$
f(x)=c+\int_{0}^{1} x^{T} \phi(t x) d t, \quad x \in \mathbb{R}^{s}, \quad c=: f(0) \in \mathbb{R},
$$


and get

$$
\begin{aligned}
\frac{\partial f}{\partial x_{j}} & =\frac{\partial}{\partial x_{j}} \int_{0}^{1} x^{T} \phi(t x) d t \\
& =\sum_{k=1}^{s} \int_{0}^{1} t x_{k} \frac{\partial \varphi_{k}}{\partial x_{j}}(t x) d t+\int_{0}^{1} \varphi_{j}(t x) d t \\
& =\sum_{k=1}^{s} \int_{0}^{1} t x_{k} \frac{\partial \varphi_{j}}{\partial x_{k}}(t x) d t+\int_{0}^{1} \varphi_{j}(t x) d t \\
& =\int_{0}^{1} t \epsilon_{j}^{T} D \phi(t x) x d t+\int_{0}^{1} \varphi_{j}(t x) d t=\int_{0}^{1} t \epsilon_{j}^{T} \frac{d}{d t} \phi(t x)+\int_{0}^{1} \varphi_{j}(t x) d t \\
& =\int_{0}^{1} t \frac{d \varphi_{j}}{d t}(t x)+\int_{0}^{1} \varphi_{j}(t x) d t \\
& =\left[t \varphi_{j}(t x)\right]_{t=0}^{1}-\int_{0}^{1} \varphi_{j}(t x) d t+\int_{0}^{1} \varphi_{j}(t x) d t=\varphi_{j}(x),
\end{aligned}
$$

that is, $\phi=\nabla f$.

Iterating (49) and passing to the Fourier transform we immediately obtain a characterization of derivative vectors of functions.

Corollary 25 A vector field $\Psi=\left[\psi_{\alpha}\right]_{|\alpha|=d}$ is the dth total derivative of a function if and only if for any $\alpha, \alpha^{\prime}$ such that $|\alpha|=\left|\alpha^{\prime}\right|=d$ and any $\beta, \beta^{\prime}$ such that $\alpha+\beta=$ $\alpha^{\prime}+\beta^{\prime}$ we have

$$
(i \xi)^{\beta} \widehat{\psi}_{\alpha}(\xi)=(i \xi)^{\beta^{\prime}} \widehat{\psi}_{\alpha^{\prime}}(\xi), \quad \xi \in \mathbb{R}^{d} .
$$

Corollary 26 For $s>1$, let be given a mask $\boldsymbol{A} \in \ell^{r_{d}}\left(\mathbb{Z}^{s}\right)$ which satisfies the spectral condition. Suppose also that the associated Taylor subdivision scheme $S_{B}$ is $C^{0}$ convergent, that its limit function $\Psi$ satisfies the conditions of Theorem 21, and that the function $\Psi_{d}$ from (30) also fulfills (50). Then $H_{A}$ is $C^{d}$-convergent.

Proof: Convergence is an immediate consequence of Theorem 21. If $\Psi_{d}$ is also an $d$ th derivative, then the functions

$$
\psi_{\alpha}(x):=\psi_{\alpha}(0)+\int_{0}^{1} \sum_{j=1}^{s} x_{j} \psi_{\alpha+\epsilon_{j}}(t x) d t, \quad|\alpha|=d-1, \quad x \in \mathbb{R}^{s},
$$

are continuous and well-defined. Choosing $\alpha, \alpha^{\prime}$ with $|\alpha|=\left|\alpha^{\prime}\right|=d-1$ and $\beta, \beta^{\prime} \neq 0$ such that $\alpha+\beta=\alpha^{\prime}+\beta^{\prime}$ as well as $j, j^{\prime} \in\{1, \ldots, s\}$ such that $\beta_{j} \neq 0$ and $\beta_{j^{\prime}}^{\prime} \neq 0$, we get that

$$
\begin{aligned}
(i \xi)^{\beta} \widehat{\psi}_{\alpha}(\xi) & =(i \xi)^{\beta-\epsilon_{j}}\left(\frac{\partial}{\partial x_{j}} \psi_{\alpha}\right)^{\wedge}(\xi)=(i \xi)^{\beta-\epsilon_{j}} \widehat{\psi}_{\alpha+\epsilon_{j}}(\xi) \\
& =(i \xi)^{\beta^{\prime}-\epsilon_{j^{\prime}}} \widehat{\psi}_{\alpha^{\prime}+\epsilon_{j^{\prime}}}(\xi)=(i \xi)^{\beta^{\prime}} \widehat{\psi}_{\alpha^{\prime}}(\xi)
\end{aligned}
$$

so that $\Psi_{d-1}=\left[\psi_{\alpha}\right]_{|\alpha|=d-1}$ is a $d-1$ st derivative. Iterating this process, we arrive at a function $\psi$ such that $D^{k} \psi=\Psi_{k}, k=1, \ldots, d$, hence the convergence is indeed a $C^{d}$ one. 
Recall that a Hermite subdivision scheme is called interpolatory if $\boldsymbol{f}_{n}(2 \alpha)=\boldsymbol{f}_{n-1}(\alpha)$, $\alpha \in \mathbb{Z}^{s}$, that is, the values computed during a certain iteration level are preserved in later iteration levels. Indeed, almost all of the "original" Hermite schemes were of this type. It was pointed out in [10, Corollary 2] that for interpolatory schemes, the spectral condition is satisfied automatically, a fact that also holds true for the multivariate situation. Hence, for any interpolatory Hermite scheme there also exists an associated Taylor scheme and convergence of the two is equivalent.

Theorem 27 If $\boldsymbol{A}$ is the mask for an interpolatory Hermite scheme, then this scheme is $C^{d}$-convergent if and only if the associated Taylor scheme is convergent with a limit as in (30) where $\Psi_{d}$ is a dth derivative, hence satisfies (50).

Proof: That the condition on the Taylor scheme is sufficient for the $C^{d}$ has already been proved, so we just have to show that $C^{d}$ convergence implies convergence of the Taylor scheme. To that end, we consider (27) to obtain for $j=1, \ldots, s,|\alpha|<d$ and $\beta \in \mathbb{Z}^{s}$ that

$$
\begin{aligned}
& 2^{-n(d-|\alpha|)} g_{j, \alpha}(\beta)=\left(T_{d} \boldsymbol{D}^{n} \boldsymbol{f}_{n}\right)_{j, \alpha} \\
& \quad=f_{n}^{(\alpha)}\left(\beta+\epsilon_{j}\right)-f_{n}^{(\alpha)}(\beta)-\sum_{k=0}^{d-|\alpha|} \frac{2^{-n k}}{k !} f_{n}^{\left(\alpha+k \epsilon_{j}\right)}(\beta) \\
& \quad=\phi_{\alpha}\left(2^{-n} \beta+2^{-n} \epsilon_{j}\right)-\phi_{\alpha}\left(2^{-n} \beta\right)-\sum_{k=0}^{d-|\alpha|} \frac{2^{-n k}}{k !} \phi_{\left(\alpha+k \epsilon_{j}\right)}\left(2^{-n} \beta\right) \\
& =D^{\alpha} \phi_{0}\left(2^{-n} \beta+2^{-n} \epsilon_{j}\right)-D^{\alpha} \phi_{0}\left(2^{-n} \beta\right)-\sum_{k=0}^{d-|\alpha|} \frac{2^{-n k}}{k !} D^{\alpha+k \epsilon_{j}} \phi_{0}\left(2^{-n} \beta\right) \\
& \quad=2^{-n(d-|\alpha|)}\left(D^{\alpha+(d-|\alpha|)} \phi_{0}(\xi)-D^{\alpha+(d-|\alpha|)} \phi_{0}\left(2^{-n} \beta\right)\right)
\end{aligned}
$$

for some $\xi \in\left[2^{-n} \beta, 2^{-n}\left(\beta+\epsilon_{j}\right)\right]$, so that indeed $\left|g_{j, \alpha}(\beta)\right| \leq \omega\left(D^{\alpha} \phi_{0}, 2^{-n}\right)$ and we have convergence to zero. For $|\alpha|=d$, (27) immediately yields that $g_{j, \alpha}(\beta)=$ $\phi_{\alpha}\left(2^{-n} \beta\right)$ which completes the proof.

\section{Remark 28}

1. The above result can be used to check the convergence of an interpolatory Hermite subdivision scheme since for stationary subdivision schemes like the Taylor scheme there exist characterizations of convergence. These imply another factorization step based on a difference operator after which convergence is characterized by a spectral radius, cf. [24]. Hence, convergence can be completely characterized for interpolatory Hermite schemes.

2. To use Theorem 27 for the construction of convergent Hermite schemes is difficult as the Taylor scheme has to satisfy two major nontrivial conditions: its limit function has to be of the form (30) and $\Psi_{d}$ has to have the gradient property (50). How to ensure these for a subdivision scheme is generally unknown 
and far from trivial. Note however, that this is a standard problem in the multivariate case where also the straightforward univariate idea of "smoothing" refinable functions provides some complications, cf. [25].

We close this section with another remark on the connection between the complete and the incomplete Taylor factorization. For simplicity, we restrict the exposition to the univariate case, the same relationship also holds true in several variables. In Theorem 21, it has been shown that convergence of the Hermite subdivision scheme $H_{\boldsymbol{A}}$ is based on the convergence on the associated Taylor subdivision scheme $S_{B}$ whose mask is obtained by $T_{d} S_{\boldsymbol{A}}=2^{-d} S_{\boldsymbol{B}} T_{d}$. Moreover, Corollary 23 tells us that in this case we automatically have $C^{d}$-convergence and Corollary 27 says that the $C^{d_{-}}$ convergence of $H_{A}$ is even equivalent to the convergence of $S_{B}$ provided that $H_{A}$ is interpolatory. In summary, convergence of $S_{B}$ can be expected to be crucial for describing the convergence of $H_{A}$.

It follows from (30) that $S_{B}$ is a subdivision scheme of rank one and that its limit function is of the form $e_{d} \Phi^{T}$ where $\Phi$ is a $d$-vector field; recall that we still restrict ourselves to the case $s=1$. The convergence of such schemes is described in [20,21], and it is equivalent to the existence of a mask $\widetilde{\boldsymbol{B}}$ such that

$$
\Delta_{d} S_{\boldsymbol{B}}=S_{\widetilde{\boldsymbol{B}}} \Delta_{d}, \quad \Delta_{d}:=\left[\begin{array}{cc}
\boldsymbol{I}_{d-1} & \mathbf{0} \\
\mathbf{0} & \Delta
\end{array}\right]
$$

and $S_{\widetilde{B}}$ is contractive. Now,

$$
\Delta_{d} T_{d} S_{\boldsymbol{A}}=\Delta_{d} S_{\boldsymbol{B}} T_{d}=S_{\widetilde{\boldsymbol{B}}} \Delta_{d} T_{d}
$$

and since

$$
\Delta_{d} T_{d}=\left[\begin{array}{cc}
\boldsymbol{I}_{d-1} & \mathbf{0} \\
\mathbf{0} & \Delta
\end{array}\right]\left[\begin{array}{ccccc}
\Delta & -1 & \ldots & -\frac{1}{(d-1) !} & -\frac{1}{d !} \\
& \Delta & \ddots & \vdots & \vdots \\
& & \ddots & -1 & \vdots \\
& & & \Delta & -1 \\
& & & & 1
\end{array}\right]=\widetilde{T}_{d}
$$

this implies that $\widetilde{T}_{d} S_{\boldsymbol{A}}=S_{\widetilde{\boldsymbol{B}}} \widetilde{T}_{d}$ and so convergence of $H_{\boldsymbol{A}}$ is also described by the existence and contractivity of $S_{\widetilde{B}}$. Based on the results from [24], the same type of relationship can also be formulated for $s>1$.

\section{Examples}

The first example from [19] considers an interpolating scheme in dimension $s=1$ with $d=1$ depending on two parameters. For this, we give the two factorizations and prove the $C^{1}$-convergence for some values of the parameters.

In the second example, from [9], we have a non interpolatory scheme and we give the complete Taylor scheme.

The third example was firstly published in [7], then simplified in [18]. It is an interpolatory scheme in dimension $s=2$ with $d=1$ derivatives whose complete Taylor scheme will be given. 


\subsection{An Interpolatory Scheme for $s=1$ and $d=1$}

We recall the interpolatory Hermite subdivision scheme $H C^{1}$, proposed by Merrien [19]. The mask has support included in $[-1,1]$ with:

$$
\boldsymbol{A}(-1)=\frac{1}{4}\left[\begin{array}{cc}
2 & 4 \lambda \\
2(1-\mu) & \mu
\end{array}\right], \boldsymbol{A}(0)=\frac{1}{2}\left[\begin{array}{ll}
2 & 0 \\
0 & 1
\end{array}\right], \boldsymbol{A}(1)=\frac{1}{4}\left[\begin{array}{cc}
2 & -4 \lambda \\
-2(1-\mu) & \mu
\end{array}\right] .
$$

For every choice of $\lambda$ and $\mu, H_{A}$ reproduces affine functions so that it satisfies the spectral condition with $p_{0}(x)=1, p_{1}(x)=x$. According to Theorem 4, there exists Taylor vector subdivision schemes $S_{\boldsymbol{B}}$ and $S_{\widetilde{B}}$ associated with $H_{\boldsymbol{A}}$.

$$
\begin{gathered}
\text { With } T_{1}=\left[\begin{array}{cc}
\Delta & -1 \\
0 & 1
\end{array}\right] \text { and } T_{1}^{*}(z)=\left[\begin{array}{cc}
z^{-1}-1 & -1 \\
0 & 1
\end{array}\right], \text { we obtain that } \\
T_{1}^{*}(z) \boldsymbol{A}^{*}(z)=\frac{1}{4}\left[\begin{array}{cc}
\left(z^{-2}-1\right) 2(1+\mu z) & 4 \lambda\left(z^{-2}-1\right)(1-z)-\mu\left(z^{-1}+z\right)-2 \\
\left(z^{-2}-1\right) 2(1-\mu) z & \left.\left.\mu\left(z^{-1}+z\right)+2\right)\right)
\end{array}\right] .
\end{gathered}
$$

The nonzero matrices of the mask of the Taylor scheme are

$$
\begin{aligned}
& \boldsymbol{B}(-2)=\left[\begin{array}{cc}
0 & 2 \lambda \\
0 & 0
\end{array}\right], \boldsymbol{B}(-1)=\left[\begin{array}{cc}
0 & -2 \lambda-\mu / 2 \\
0 & \mu / 2
\end{array}\right], \boldsymbol{B}(0)=\left[\begin{array}{cc}
1 & -2 \lambda \\
0 & 1
\end{array}\right] \text { and } \boldsymbol{B}(1)= \\
& {\left[\begin{array}{cc}
\mu & 2 \lambda+\mu / 2 \\
1-\mu & 1-\mu / 2
\end{array}\right] .}
\end{aligned}
$$

Similarly with $\widetilde{T}_{1}=\left[\begin{array}{cc}\Delta & -1 \\ 0 & \Delta\end{array}\right]$ the nonzero matrices of the mask of the complete Taylor scheme are found to be

$$
\widetilde{\boldsymbol{B}}(0)=\left[\begin{array}{cc}
1 & 2 \lambda \\
(1-\mu) & \mu / 2
\end{array}\right], \quad \widetilde{\boldsymbol{B}}(1)=\left[\begin{array}{cc}
\mu & -\mu / 2-2 \lambda \\
\mu-1 & 1-\mu / 2
\end{array}\right] .
$$

For $\lambda>-1 / 8$ and $\mu<1$, we define a vector norm by $\|v\|:=\left\|P^{-1} v\right\|_{2}$, where $\|\cdot\|_{2}$ is the usual Euclidean vector norm and $P:=\left[\begin{array}{cc}1 & \sqrt{\frac{1-\mu}{1+8 \lambda}} \\ 0 & 2 \sqrt{\frac{1-\mu}{1+8 \lambda}}\end{array}\right]$. The corresponding matrix operator norm is given by $\|M\|:=\left\|P^{-1} M P\right\|_{2}$, where $\|N\|_{2}:=\sqrt{\rho\left(N^{T} N\right)}$ is the spectral norm of a matrix $N$. Then

$$
\begin{aligned}
& P^{-1} \tilde{\boldsymbol{B}}(0) P=\frac{1}{2}\left[\begin{array}{cc}
1+\mu & \sqrt{(1-\mu)(1+8 \lambda)} \\
\sqrt{(1-\mu)(1+8 \lambda)} & 1
\end{array}\right] \\
& P^{-1} \tilde{\boldsymbol{B}}(1) P=\frac{1}{2}\left[\begin{array}{cc}
1+\mu & -\sqrt{(1-\mu)(1+8 \lambda)} \\
-\sqrt{(1-\mu)(1+8 \lambda)} & 1
\end{array}\right]
\end{aligned}
$$

are symmetric. The eigenvalues of $\tilde{\boldsymbol{B}}(i)_{i=0,1}$ or of $P^{-1} \tilde{\boldsymbol{B}}(i) P$ are

$$
\begin{aligned}
& \ell_{1}=\frac{1}{4}\left(2+\mu+\sqrt{(2-\mu)^{2}+32 \lambda(1-\mu)}\right) \\
& \ell_{2}=\frac{1}{4}\left(2+\mu-\sqrt{(2-\mu)^{2}+32 \lambda(1-\mu)}\right) .
\end{aligned}
$$

Since $P^{-1} \tilde{\boldsymbol{B}}(i) P$ are symmetric, the eigenvalues are real with $\ell_{2}<\ell_{1}$. Now for $\mu \in$ $[-2,1)$ and $\lambda \in(-1 / 8,0)$ we find $\ell_{1}<\left(2+\mu+\sqrt{(2-\mu)^{2}}\right) / 4=1$ and $\ell_{2}>(2+\mu-$ 
$\left.\sqrt{(2-\mu)^{2}}\right) / 4=\mu / 2 \geq-1$. Thus $\rho:=\|\tilde{\boldsymbol{B}}(i)\|=\max \left\{\left|\ell_{1}\right|,\left|\ell_{2}\right|\right\}<1$ and we have shown that $S_{\tilde{B}}$ is contractive. We can extend the result to $(\lambda, \mu) \in[-1 / 8,0) \times[-2,1)$ and we can deduce the $C^{1}$-convergence of $H_{\boldsymbol{A}}$ for $(\lambda, \mu) \in[-1 / 8,0) \times(-2,1)$.

More precisely, the contractivity of $S_{\tilde{B}}$ is obtained if the joint spectral radius of $\{\tilde{\boldsymbol{B}}(0), \tilde{\boldsymbol{B}}(1)\}$ is less than 1 and following [5], the $C^{1}$-convergence of $H_{\boldsymbol{A}}$ is obtained if and only if $-1 / 2<\lambda<0$ and $1+\max \left(\frac{1}{2 \lambda}, \frac{-3}{1+2 \lambda}\right)<\mu<1$.

\subsection{A Non Interpolatory Scheme for $s=1$ and $d=1$}

We build the de Rham transform [9] of the previous scheme $H_{\boldsymbol{A}}$ with support in $[-2,1]$. The respective non zero matrices are

$$
\begin{gathered}
\frac{1}{8}\left[\begin{array}{cc}
2+4 \lambda(1-\mu) & 4 \lambda+2 \lambda \mu \\
4-2 \mu-2 \mu^{2} & \mu^{2}+8 \lambda(1-\mu)
\end{array}\right], \frac{1}{8}\left[\begin{array}{cc}
6-4 \lambda(1-\mu) & 8 \lambda-2 \lambda \mu \\
4-2 \mu-2 \mu^{2} & 2 \mu+\mu^{2}-8 \lambda(1-\mu)
\end{array}\right], \\
\frac{1}{8}\left[\begin{array}{cc}
6-4 \lambda(1-\mu) & -8 \lambda+2 \lambda \mu \\
-4+2 \mu+2 \mu^{2} & 2 \mu+\mu^{2}-8 \lambda(1-\mu)
\end{array}\right], \frac{1}{8}\left[\begin{array}{cc}
2+4 \lambda(1-\mu) & -4 \lambda-2 \lambda \mu \\
-4+2 \mu+2 \mu^{2} & \mu^{2}+8 \lambda(1-\mu)
\end{array}\right] .
\end{gathered}
$$

We notice that $H_{\boldsymbol{A}}$ satisfies the spectral condition with $p_{0}(x)=1, p_{1}(x)=x-1 / 2$. A complete Taylor scheme $S_{\widetilde{\boldsymbol{B}}}$ is associated with $H_{\boldsymbol{A}}$. The nonzero matrices $\widetilde{\boldsymbol{B}}$ of the mask are $\widetilde{\boldsymbol{B}}(\alpha), \alpha \in[-1,1]$ with

$$
\begin{aligned}
\widetilde{\boldsymbol{B}}(-1) & =\frac{1}{4}\left[\begin{array}{cc}
2+4 \lambda(1-\mu) & 2 \lambda(2+\mu) \\
4-2 \mu-2 \mu^{2} & \mu^{2}+8 \lambda(1-\mu)
\end{array}\right], \\
\widetilde{\boldsymbol{B}}(0) & =\frac{1}{4}\left[\begin{array}{cc}
2 \mu+2 \mu^{2}-8 \lambda(1-\mu) & -4 \lambda(1-\mu)-\mu^{2} \\
0 & 2 \mu-16 \lambda(1-\mu)
\end{array}\right], \\
\widetilde{\boldsymbol{B}}(1) & =\frac{1}{4}\left[\begin{array}{cc}
-2+4 \lambda(1-\mu)+2 \mu+2 \mu^{2} & -6 \lambda \mu-\mu^{2}-2 \mu+2 \\
-4+2 \mu+2 \mu^{2} & 4-2 \mu-\mu^{2}+8 \lambda(1-\mu)
\end{array}\right] .
\end{aligned}
$$

\subsection{An Interpolatory Scheme for $s=2$ and $d=1$}

The $H R C^{1}$-Algorithm has a mask supported in $[-1,1]^{2}$. For $\eta_{1}, \eta_{2} \in\{ \pm 1\}$

$$
\begin{array}{ccc}
\boldsymbol{A}(0,0)=\boldsymbol{D}=\left[\begin{array}{ccc}
1 & 0 & 0 \\
0 & \frac{1}{2} & 0 \\
0 & 0 & \frac{1}{2}
\end{array}\right], & \boldsymbol{A}\left(\eta_{1}, 0\right)=\left[\begin{array}{ccc}
\frac{1}{2} & -\eta_{1} \lambda & 0 \\
-\eta_{1} \frac{1-\mu}{2} & \frac{\mu}{4} & 0 \\
0 & 0 & \frac{1}{4}
\end{array}\right], \\
\boldsymbol{A}\left(0, \eta_{2}\right)=\left[\begin{array}{ccc}
\frac{1}{2} & 0 & -\eta_{2} \lambda \\
0 & \frac{1}{4} & 0 \\
-\eta_{2} \frac{1-\mu}{2} & 0 & \frac{\mu}{4}
\end{array}\right], & \boldsymbol{A}\left(\eta_{1}, \eta_{2}\right)\left[\begin{array}{ccc}
\frac{1}{4} & -\eta_{1} \frac{\lambda}{2} & -\eta_{2} \frac{\lambda}{2} \\
-\eta_{1} \frac{1-\mu}{1-\mu} & \frac{\mu}{8} & \eta_{1} \eta_{2} \frac{\mu}{8} \\
-\eta_{2} \frac{1-\mu}{4} & \eta_{1} \eta_{2} \frac{\mu}{8} & \frac{\mu}{8}
\end{array}\right] .
\end{array}
$$

We have simplified the construction of [7] and it depends on two parameters $\lambda$ and $\mu$ only. For $\lambda=-1 / 8, \mu=-1$ we obtain the Sibson-Thomson interpolant on every initial square, see [27]. In this case, the $H R C^{1}$-interpolant is a $C^{1}$ piecewise quadratic consisting of 16 individual pieces. It was also shown that the $H R C^{1}$-algorithm is exact for bilinear functions for any value of $\lambda$ and $\mu$ which implies that the spectral property is satisfied with $p_{(0,0)}(x, y)=1, p_{(1,0)}(x, y)=x$ and $p_{(0,1)}(x, y)=y$. Thus, a Taylor 
subdivision scheme $S_{\widetilde{\boldsymbol{B}}} \in \ell^{6 \times 6}\left(\mathbb{Z}^{2}\right)$ is associated with $H_{\boldsymbol{A}}$. The nonzero matrices $\widetilde{\boldsymbol{B}}$ of the mask are:

$$
\begin{aligned}
& \widetilde{\boldsymbol{B}}(1,1)=\left[\begin{array}{cccccc}
\frac{\mu}{4} & -\frac{\lambda}{2}-\frac{\mu}{8} & -\frac{\lambda}{2}+\frac{\mu}{8} & 0 & 0 & 0 \\
\frac{\mu-1}{4} & -\frac{\mu}{8}+\frac{1}{4} & \frac{\mu}{8} & 0 & 0 & 0 \\
\frac{\mu-1}{4} & \frac{\mu}{8} & \frac{\mu}{8} & 0 & -\frac{\mu-1}{4} & 0 \\
0 & 0 & 0 & \frac{\mu}{4} & -\frac{\lambda}{2}+\frac{\mu}{8} & -\frac{\lambda}{2}-\frac{\mu}{8} \\
0 & 0 & -\frac{\mu-1}{4} & \frac{\mu-1}{4} & \frac{\mu}{8} & \frac{\mu}{8} \\
0 & 0 & 0 & \frac{\mu-1}{4} & \frac{\mu}{8} & -\frac{\mu}{8}+\frac{1}{4}
\end{array}\right], \\
& \widetilde{\boldsymbol{B}}(0,1)=\left[\begin{array}{cccccc}
\frac{1}{4} & \frac{\lambda}{2} & -\frac{\lambda}{2} & 0 & 0 & 0 \\
-\frac{\mu-1}{4} & \frac{\mu}{8} & -\frac{\mu}{8} & 0 & 0 & 0 \\
\frac{\mu-1}{4} & -\frac{\mu}{8} & \frac{\mu}{8} & 0 & -\frac{\mu-1}{4} & 0 \\
0 & 0 & 0 & \frac{\mu}{2} & 0 & -\lambda-\frac{\mu}{4} \\
0 & 0 & 0 & 0 & \frac{1}{4} & 0 \\
0 & 0 & 0 & \frac{\mu-1}{2} & 0 & -\frac{\mu}{4}+\frac{1}{2}
\end{array}\right] \\
& \widetilde{\boldsymbol{B}}(-1,1)=\left[\begin{array}{cccccc}
0 & 0 & 0 & 0 & 0 & 0 \\
0 & 0 & 0 & 0 & 0 & 0 \\
0 & 0 & 0 & 0 & 0 & 0 \\
0 & 0 & 0 & \frac{\mu}{4} & \frac{\lambda}{2}-\frac{\mu}{8} & -\frac{\lambda}{2}-\frac{\mu}{8} \\
0 & 0 & 0 & -\frac{\mu-1}{4} & \frac{\mu}{8} & -\frac{\mu}{8} \\
0 & 0 & 0 & \frac{\mu-1}{4} & -\frac{\mu}{8} & -\frac{\mu}{8}+\frac{1}{4}
\end{array}\right] \\
& \widetilde{\boldsymbol{B}}(1,0)=\left[\begin{array}{cccccc}
\frac{\mu}{2} & -\lambda-\frac{\mu}{4} & 0 & 0 & 0 & 0 \\
\frac{\mu-1}{2} & -\frac{\mu}{4}+\frac{1}{2} & 0 & 0 & 0 & 0 \\
0 & 0 & \frac{1}{4} & 0 & 0 & 0 \\
0 & 0 & 0 & \frac{1}{4} & -\frac{\lambda}{2} & \frac{\lambda}{2} \\
0 & 0 & -\frac{\mu-1}{4} & \frac{\mu-1}{4} & \frac{\mu}{8} & -\frac{\mu}{8} \\
0 & 0 & 0 & -\frac{\mu-1}{4} & -\frac{\mu}{8} & \frac{\mu}{8}
\end{array}\right] \\
& \widetilde{\boldsymbol{B}}(0,0)=\left[\begin{array}{cccccc}
\frac{1}{2} & \lambda & 0 & 0 & 0 & 0 \\
-\frac{\mu-1}{2} & \frac{\mu}{4} & 0 & 0 & 0 & 0 \\
0 & 0 & \frac{1}{4} & 0 & 0 & 0 \\
0 & 0 & 0 & \frac{1}{2} & 0 & \lambda \\
0 & 0 & 0 & 0 & \frac{1}{4} & 0 \\
0 & 0 & 0 & -\frac{\mu-1}{2} & 0 & \frac{\mu}{4}
\end{array}\right] \\
& \widetilde{\boldsymbol{B}}(-1,0)=\left[\begin{array}{cccccc}
0 & 0 & 0 & 0 & 0 & 0 \\
0 & 0 & 0 & 0 & 0 & 0 \\
0 & 0 & 0 & 0 & 0 & 0 \\
0 & 0 & 0 & \frac{1}{4} & \frac{\lambda}{2} & \frac{\lambda}{2} \\
0 & 0 & 0 & -\frac{\mu-1}{4} & \frac{\mu}{8} & \frac{\mu}{8} \\
0 & 0 & 0 & -\frac{\mu-1}{4} & \frac{\mu}{8} & \frac{\mu}{8}
\end{array}\right]
\end{aligned}
$$




$$
\begin{gathered}
\widetilde{\boldsymbol{B}}(1,-1)=\left[\begin{array}{cccccc}
\frac{\mu}{4} & -\frac{\lambda}{2}-\frac{\mu}{8} & \frac{\lambda}{2}-\frac{\mu}{8} & 0 & 0 & 0 \\
\frac{\mu-1}{4} & -\frac{\mu}{8}+\frac{1}{4} & -\frac{\mu}{8} & 0 & 0 & 0 \\
-\frac{\mu-1}{4} & -\frac{\mu}{8} & \frac{\mu}{8} & 0 & 0 & 0 \\
0 & 0 & 0 & 0 & 0 & 0 \\
0 & 0 & 0 & 0 & 0 & 0 \\
0 & 0 & 0 & 0 & 0 & 0
\end{array}\right] \\
\widetilde{B}(0,-1)=\left[\begin{array}{cccccc}
\frac{1}{4} & \frac{\lambda}{2} & \frac{\lambda}{2} & 0 & 0 & 0 \\
-\frac{\mu-1}{4} & \frac{\mu}{8} & \frac{\mu}{8} & 0 & 0 & 0 \\
-\frac{\mu-1}{4} & \frac{\mu}{8} & \frac{\mu}{8} & 0 & 0 & 0 \\
0 & 0 & 0 & 0 & 0 & 0 \\
0 & 0 & 0 & 0 & 0 & 0 \\
0 & 0 & 0 & 0 & 0 & 0
\end{array}\right] .
\end{gathered}
$$

In [18], sufficient conditions are given to obtain the $C^{1}$-convergence of the scheme for different values of the parameters.

Two examples of interpolatory schemes for $s=2$ and $d=2$ were also proposed in [6].

\section{References}

[1] A. S. Cavaretta, W. Dahmen, and C. A. Micchelli, Stationary subdivision, Memoirs of the AMS, vol. 93 (453), Amer. Math. Soc., 1991.

[2] CoCoATeam, CoCoA: a system for doing Computations in Commutative Algebra, Available at http://cocoa.dima.unige.it.

[3] D. Cox, J. Little, and D. O'Shea, Ideals, varieties and algorithms, 2. ed., Undergraduate Texts in Mathematics, Springer-Verlag, 1996.

[4] W. Dahmen and C. A. Micchelli, Biorthogonal wavelet expansion, Constr. Approx. 13 (1997), 294-328.

[5] S. Dubuc, Scalar and Hermite subdivision schemes, Appl. Comput. Harmonic Anal. 21 (2006), 376-394.

[6] S. Dubuc, B. Han, J.-L. Merrien, and Q. Mo, Dyadic $C^{2}$ Hermite interpolation on a square mesh, Comp. Aided Geom. Design 22 (2005), 727-752.

[7] S. Dubuc and J.-L. Merrien, Dyadic Hermite interpolation on a rectangular mesh, Adv. Comput. Math. 10 (1999), 342-365.

[8] _ Convergent vector and Hermite subdivision schemes, Constr. Approx. 23 (2006), 1-22.

[9]___ Rham Transform of a Hermite Subdivision Scheme, Approximation Theory XII, San Antonio 2007, M. Neamtu and L.L. Schumaker eds, 2008, Nashboro Press, Nashville TN, 121-132.

[10] _ Hermite subdivision schemes and Taylor polynomials, Constr. Approx. 29 (2009), 219-245. 
[11] N. Dyn, D. Levin, Analysis of Hermite-type subdivision schemes, (C. K. Chui and L. L. Schumaker Eds.), Approximation Theory VIII. Vol 2: Wavelets and Multilevel Approximation (College Station, TX, 1995). World Sci., River Edge, NJ, 1995, pp. 117-124.

[12] N. Dyn, D. Levin, Analysis of Hermite-interpolatory subdivision schemes, (S. Dubuc et G. Deslauriers, Eds.), Spline Functions and the Theory of Wavelets, Amer. Math. Soc., Providence R. I., 1999, pp. 105-113.

[13] N. Dyn and D. Levin, Subdivision schemes in geometric modelling, Acta Numerica 11 (2002), 73-144.

[14] O. Föllinger, Laplace-, Fourier- und z-Transformation, Hüthig, 2000.

[15] C. Gasquet and P. Witomski, Fourier analysis and applications. Filtering, numerical computation, wavelets, Texts in Applied Mathematics, vol. 30, Springer, 1998.

[16] B. Han, Vector cascade algorithms and refinable functions in sobolev spaces, J. Approx. Theory 124 (2003), 44-88.

[17] B. Han, Th. Yu, and Y. Xue, Noninterpolatory Hermite subdivision schemes, Math. Comp. 74 (2005), 1345-1367.

[18] T. Lyche and J.-L. Merrien, $C^{1}$ Hermite subdivision with shape constrains on a rectangular mesh, BIT 46 (2006), 831-859.

[19] J.-L. Merrien, A family of Hermite interpolants by bisection algorithms, Numer. Algo. 2 (1992), 187-200.

[20] C. A. Micchelli and T. Sauer, Regularity of multiwavelets, Advances Comput. Math. 7 (1997), no. 4, 455-545.

[21] C. A. Micchelli and T. Sauer, On vector subdivision, Math. Z. 229 (1998), 621674.

[22] H. M. Möller and T. Sauer, Multivariate refinable functions of high approximation order via quotient ideals of Laurent polynomials, Advances Comput. Math 20 (2004), 205-228.

[23] T. Sauer, Polynomial interpolation, ideals and approximation order of refinable functions, Proc. Amer. Math. Soc. 130 (2002), 3335-3347.

[24] __ Stationary vector subdivision - quotient ideals, differences and approximation power, Rev. R. Acad. Cien. Serie A. Mat. 96 (2002), 257-277.

[25] _ How to generate smoother refinable functions from given ones, Modern Developments in Multivariate Approximation (W. Haussmann, K. Jetter, M. Reimer, and J. Stöckler, eds.), International Series of Numerical Mathematics, vol. 145, Birkhäuser, 2003, pp. 279-294.

[26] _ Differentiability of multivariate refinable functions and factorization, Advances Comput. Math. 25 (2006), 211-235. 
[27] R. Sibson and G. D. Thomson, A seamed quadratic element for contouring, The Computer Journal 24 (1981), 378-382.

[28] M. Vetterli and J. Kovačević, Wavelets and subband coding, Prentice Hall, 1995. 